ITEP-TH-04/21

\title{
Kadomtsev-Petviashvili hierarchies of types B and C
}

\author{
A. Zabrodin * \\ February 2021 \\ Dedicated to the memory of S.M. Natanzon
}

\begin{abstract}
This is a short review of the Kadomtsev-Petviashvili hierarchies of types B and C. The main objects are the $L$-operator, the wave operator, the auxiliary linear problems for the wave function, the bilinear identity for the wave function and the tau-function. All of them are discussed in the paper. The connections with the usual Kadomtsev-Petviashvili hierarchy (of the type A) are clarified. Examples of soliton solutions and the dispersionless limit of the hierarchies are also considered.
\end{abstract}

\section{Contents}

1 Introduction $\quad 2$

2 The KP hierarchy

3 The BKP hierarchy 5

3.1 The Lax operator and the wave operator . . . . . . . . . . . 5

3.2 The wave function and the tau-function . . . . . . . . . . . . 7

3.3 The BKP hierarchy and the KP hierarchy . . . . . . . . . . . . . . . 11

3.4 Examples: soliton solutions . . . . . . . . . . . . 12

3.4.1 One-soliton solution . . . . . . . . . . . . . . 12

3.4.2 Multi-soliton solutions . . . . . . . . . . . . . 13

*Skolkovo Institute of Science and Technology, 143026, Moscow, Russia and Institute of Biochemical Physics, Kosygina str. 4, 119334, Moscow, Russia and ITEP NRC KI, 25 B.Cheremushkinskaya, Moscow 117218, Russia; e-mail: zabrodin@itep.ru 
4.1 The CKP equation and the hierarchy . . . . . . . . . . 14

4.2 The wave function and the tau-function . . . . . . . . . 14

4.3 The equation for the tau-function . . . . . . . . . . 18

4.4 The CKP hierarchy and the KP hierarchy . . . . . . . . . . . 19

4.5 Examples: soliton solutions . . . . . . . . . . . . . . . . . 21

4.5 .1 One-soliton solution . . . . . . . . . . . . . . 21

4.5.2 Multi-soliton solutions . . . . . . . . . . . . . . . 22

5 Dispersionless limit of the BKP and CKP hierarchies 22

Acknowledgments 24

References $\quad 24$

\section{Introduction}

In the papers [1, 2, 3] infinite integrable hierarchies of partial differential equations with $\mathrm{O}(\infty)$ and $\mathrm{Sp}(\infty)$ symmetry were introduced. They can be called the KadomtsevPetviashvili hierarchies of type B (BKP) and C (CKP). The BKP (respectively, CKP) hierarchy was also discussed in [4, 5, 6, 7]. (respectively, [7, 8, 9, 10, 11]).

As is pointed out in [3], the general solutions to the BKP and CKP hierarchies depend on functional parameters in two variables. In a certain sense to be clarified below in the main text these hierarchies can be regarded as restrictions of the well known KadomtsevPetviashvili (KP) hierarchy. In a nutshell, this can be made more precise as follows. Let $\mathcal{X}_{\mathrm{KP}}$ be the moduli space of solutions to the KP hierarchy (according to Segal and Wilson, it is an infinite dimensional Grassmann manifold). The modili spaces of solutions to the $\mathrm{BKP}$ and CKP hierarchies are submanifolds of $\mathcal{X}_{\mathrm{KP}}: \mathcal{X}_{\mathrm{BKP}} \subset \mathcal{X}_{\mathrm{KP}}, \mathcal{X}_{\mathrm{CKP}} \subset \mathcal{X}_{\mathrm{KP}}$, and the "even" time evolution (i.e., the evolution with respect to the times $t_{2 k}, k \geq 1$ ) is frozen.

This paper is a short review of the BKP and CKP hierarchies. We discuss the main objects and notions related to them: the $L$-operator, the wave operator, the auxiliary linear problems for the wave function, the bilinear identity for the wave function and the tau-function. The tau-function satisfies certain equations (the Hirota equations) which are bilinear in the BKP case and have a more complicated structure in the CKP case. The connection between tau-functions of the KP, BKP and CKP hierarchies is clarified.

As examples of solutions, we give explicit formulas for soliton solutions. BKP and CKP $N$-solitons are specializations of $2 N$ soliton solutions of the KP hierarchy. As is known, soliton solutions are degenerations of more general quasi-periodic (algebrogeometric) solutions. According to the Krichever's construction [12], any smooth algebraic curve with some additional data provides a quasi-periodic solution. The quasiperiodic solutions of the BKP hierarchy were constructed in [13], see also [14]. A detailed 
discussion of quasi-periodic solutions to the CKP hierarchy can be found in [11]. The corresponding algebraic curves should admit a holomorphic involution with two fixed points. Double-periodic in the complex plane (elliptic) solutions were studied in [15, 16] for BKP and [11] for CKP.

We also discuss the zero dispersion limit of the BKP and CKP hierarchies which appears to be the same for both of them. In the dispersionless limit, the operator $\partial_{x}$ entering the pseudo-differential Lax operator is replaced by a commuting variable $p$, the Lax operator becomes a commuting function (a Laurent series) and the commutator is replaced by the Poisson bracket $\{p, x\}=1$.

\section{The KP hierarchy}

Here we briefly recall the main notions related to the KP hierarchy. The set of independent variables ("times") is $\mathbf{t}=\left\{t_{1}, t_{2}, t_{3}, \ldots\right\}$. It is convenient to set $t_{1}=x+$ const, so that the vector fields $\partial_{t_{1}}$ and $\partial_{x}$ are identical: $\partial_{t_{1}}=\partial_{x}$. The main object is the $L$-operator which is a pseudo-differential operator of the form

$$
L=\partial_{x}+u_{1} \partial_{x}^{-1}+u_{2} \partial_{x}^{-2}+\ldots
$$

with no restrictions on the coefficient functions $u_{i}$. The coefficient functions depend on $x$ and on all the times: $u_{i}=u_{i}(x, \mathbf{t})$. Together with the Lax operator, it is convenient to introduce the wave operator (or dressing operator)

$$
W=1+\xi_{1} \partial_{x}^{-1}+\xi_{2} \partial_{x}^{-2}+\ldots
$$

such that

$$
L=W \partial_{x} W^{-1}
$$

(the latter equality is interpreted as "dressing" of the operator $\partial_{x}$ by $W$ ). Clearly, there is a freedom in the definition of the wave operator: it can be multiplied from the right by any pseudo-differential operator with constant coefficients.

The functions $u_{i}(x, 0)$ are initial conditions for the time evolution $u_{i}(x, 0) \rightarrow u_{i}(x, \mathbf{t})$ which is generated by the Lax equations of the KP hierarchy:

$$
\partial_{t_{k}} L=\left[B_{k}, L\right], \quad B_{k}=\left(L^{k}\right)_{+}, \quad k=1,2,3, \ldots,
$$

where $(\ldots)_{+}$means the differential part of a pseudo-differential operator (i.e. terms with non-negative powers of $\partial_{x}$ ). In particular, $B_{1}=\partial_{x}$ and $B_{2}=\partial_{x}^{2}+2 u_{1}$. Since $B_{1}=\partial_{x}$, it follows from (2.4) that the evolution in the time $t_{1}$ is simply the shift of $x$, i.e. the solutions depend on $x+t_{1}$.

An equivalent formulation of the hierarchy is through the zero curvature (ZakharovShabat) equations

$$
\partial_{t_{l}} B_{k}-\partial_{t_{k}} B_{l}+\left[B_{k}, B_{l}\right]=0 .
$$

The equivalence of the Lax and Zakharov-Shabat formulations was proved in [20]. The famous KP equation for $u_{1}$ is obtained from (2.5) at $k=2, l=3$. 
The Lax equations and the zero curvature equations are compatibility conditions of the auxiliary linear problems

$$
\partial_{t_{k}} \psi=B_{k} \psi, \quad L \psi=z \psi
$$

for the formal wave function

$$
\psi=\psi(x, \mathbf{t}, z)=W e^{x z+\xi(\mathbf{t}, z)},
$$

where $W$ is the wave operator (2.2), $z$ is the spectral parameter and

$$
\xi(\mathbf{t}, z)=\sum_{k \geq 1} t_{k} z^{k}
$$

(it is implied that the operator $\partial_{x}^{-1}$ acts to the exponential function as $\partial_{x}^{-1} e^{x z}=z^{-1} e^{x z}$ ). One can also introduce the adjoint (dual) wave function

$$
\psi^{\dagger}=\psi^{\dagger}(x, \mathbf{t}, z)=\left(W^{\dagger}\right)^{-1} e^{-x z-\xi(\mathbf{t}, z)}
$$

where $\dagger$ means the formal adjoint defined by the rule $\left(f(x) \circ \partial_{x}^{n}\right)^{\dagger}=\left(-\partial_{x}\right)^{n} \circ f(x)$. It can be shown that the adjoint wave function satisfies the adjoint linear equations

$$
-\partial_{t_{k}} \psi^{\dagger}=B_{k}^{\dagger} \psi^{\dagger}
$$

The tau-function $\tau^{\mathrm{KP}}(x, \mathbf{t})$ of the KP hierarchy is consistently introduced by the equations

$$
\begin{gathered}
\psi(x, \mathbf{t}, z)=e^{x z+\xi(\mathbf{t}, z)} \frac{\tau^{\mathrm{KP}}\left(x, \mathbf{t}-\left[z^{-1}\right]\right)}{\tau^{\mathrm{KP}}(x, \mathbf{t})}, \\
\psi^{\dagger}(x, \mathbf{t}, z)=e^{-x z-\xi(\mathbf{t}, z)} \frac{\tau^{\mathrm{KP}}\left(x, \mathbf{t}+\left[z^{-1}\right]\right)}{\tau^{\mathrm{KP}}(x, \mathbf{t})}
\end{gathered}
$$

where we have used the standard notation

$$
\mathbf{t}+j\left[z^{-1}\right]=\left\{t_{1}+\frac{j}{z}, t_{2}+\frac{j}{2 z^{2}}, t_{3}+\frac{j}{3 z^{3}}, \ldots\right\}, \quad j \in \mathbb{Z}
$$

The wave functions satisfy the bilinear equation [4]

$$
\oint_{C_{\infty}} \psi(x, \mathbf{t}, z) \psi^{\dagger}\left(x, \mathbf{t}^{\prime}, z\right) \frac{d z}{2 \pi i}=0
$$

for all $\mathbf{t}, \mathbf{t}^{\prime}$. Here $C_{\infty}$ is a contour surrounding $\infty$ (a big circle of radius $R \rightarrow \infty$ ). Using (2.11), (2.12), one can rewrite (2.13) as the following bilinear relation for the tau-function:

$$
\oint_{C_{\infty}} e^{\xi\left(\mathbf{t}-\mathbf{t}^{\prime}, z\right)} \tau\left(x, \mathbf{t}-\left[z^{-1}\right]\right) \tau\left(x, \mathbf{t}^{\prime}+\left[z^{-1}\right]\right) \frac{d z}{2 \pi i}=0 .
$$

This is the generating equation for all differential equations of the KP hierarchy. A direct consequence of the bilinear relation (2.13) is the Hirota-Miwa equation for the tau-function of the KP hierarchy

$$
\begin{aligned}
& \left(z_{1}-z_{2}\right) \tau^{\mathrm{KP}}\left(x, \mathbf{t}-\left[z_{1}^{-1}\right]-\left[z_{2}^{-1}\right]\right) \tau^{\mathrm{KP}}\left(x, \mathbf{t}-\left[z_{3}^{-1}\right]\right) \\
& +\left(z_{2}-z_{3}\right) \tau^{\mathrm{KP}}\left(x, \mathbf{t}-\left[z_{2}^{-1}\right]-\left[z_{3}^{-1}\right]\right) \tau^{\mathrm{KP}}\left(x, \mathbf{t}-\left[z_{1}^{-1}\right]\right) \\
& \quad+\left(z_{3}-z_{1}\right) \tau^{\mathrm{KP}}\left(x, \mathbf{t}-\left[z_{1}^{-1}\right]-\left[z_{3}^{-1}\right]\right) \tau^{\mathrm{KP}}\left(x, \mathbf{t}-\left[z_{2}^{-1}\right]\right)=0
\end{aligned}
$$


It is a generating equation for the differential equations of the hierarchy. In the limit $z_{3} \rightarrow \infty$ it becomes the equation

$$
\begin{aligned}
& \partial_{x} \log \frac{\tau^{\mathrm{KP}}\left(x, \mathbf{t}+\left[z_{1}^{-1}\right]-\left[z_{2}^{-1}\right]\right)}{\tau^{\mathrm{KP}}(x, \mathbf{t})} \\
& =\left(z_{2}-z_{1}\right)\left(\frac{\tau^{\mathrm{KP}}\left(x, \mathbf{t}+\left[z_{1}^{-1}\right]\right) \tau^{\mathrm{KP}}\left(x, \mathbf{t}-\left[z_{2}^{-1}\right]\right)}{\tau^{\mathrm{KP}}(x, \mathbf{t}) \tau^{\mathrm{KP}}\left(x, \mathbf{t}+\left[z_{1}^{-1}\right]-\left[z_{2}^{-1}\right]\right)}-1\right) .
\end{aligned}
$$

The tau-function $\tilde{\tau}(x, \mathbf{t})=e^{\ell(x, \mathbf{t})} \tau(x, \mathbf{t})$, where $\ell(x, \mathbf{t})=\gamma_{0}+\gamma_{1} x+\sum_{k \geq 1} \gamma_{k} t_{k}$ is a linear function of the times, satisfies the same bilinear equations. We say that the tau-functions which differ by a factor of the form $e^{\ell(x, \mathbf{t})}$ are equivalent.

\section{The BKP hierarchy}

Here we present the main formulas related to the BKP hierarchy with some details. The main reference is [2], see also [4, 5, 6, 7].

\subsection{The Lax operator and the wave operator}

The set of independent variables ("times") is $\mathbf{t}_{\mathrm{o}}=\left\{t_{1}, t_{3}, t_{5}, \ldots\right\}$. They are indexed by positive odd numbers. As in the KP hierarchy, we set $t_{1}=x+$ const, so that the vector fields $\partial_{t_{1}}$ and $\partial_{x}$ are identical: $\partial_{t_{1}}=\partial_{x}$. The main object is the $L$-operator which is a pseudo-differential operator of the form

$$
L=\partial_{x}+u_{1} \partial_{x}^{-1}+u_{2} \partial_{x}^{-2}+\ldots
$$

with the constraint

$$
L^{\dagger}=-\partial_{x} L \partial_{x}^{-1}
$$

Unlike in the case of a reduction, when only a finite number of the coefficient functions $u_{i}$ remain independent, the constraint (3.2) implies that there are still infinitely many independent coefficients functions. As we shall see soon, the constraint (3.2) is invariant under the "odd" flows $t_{1}, t_{3}, t_{5}, \ldots$ of the KP hierarchy, so the BKP hierarchy can be regarded as a sub-hierarchy (a restriction) of the KP one with the "even" times frozen.

It is instructive to reformulate the constraint (3.2) in terms of the wave operator $W$ (2.2) such that $L=W \partial_{x} W^{-1}$. The constraint (3.2) implies that $W^{\dagger} \partial_{x} W$ commutes with $\partial_{x}$, i.e., it is a pseudo-differential operator with constant coefficients. The freedom in the definition of the wave operator can be fixed by demanding that $W^{\dagger} \partial_{x} W=\partial_{x}$, i.e.

$$
W^{\dagger}=\partial_{x} W^{-1} \partial_{x}^{-1}
$$

The Lax equations of the hierarchy are the same as (2.4) but with odd indices:

$$
\partial_{t_{k}} L=\left[B_{k}, L\right], \quad B_{k}=\left(L^{k}\right)_{+}, \quad k=1,3,5, \ldots
$$


The constraint (3.2) is equivalent to the condition that the differential operators $B_{k}$ satisfy $B_{k} \cdot 1=0$ (for odd $k$ ), i.e., that they have the form

$$
B_{k}=\partial_{x}^{k}+\sum_{j=1}^{k-2} b_{k, j} \partial_{x}^{j}
$$

with $b_{k, 0}=0$. Indeed, if (3.2) is satisfied, then $L^{n} \partial_{x}^{-1}=-\partial_{x}^{-1} L^{\dagger n}$ for odd $n$. On the other hand, $\left(L^{n} \partial_{x}^{-1}\right)^{\dagger}=-\partial_{x}^{-1} L^{\dagger n}=L^{n} \partial_{x}^{-1}$ which implies that the coefficient in front of $\partial_{x}^{0}$ in $L^{n}$ vanishes: $b_{n, 0}=0$ for odd $n$. Conversely, assuming that $b_{n, 0}=0$ for all odd $n$, we shall prove that $R=\partial_{x}^{-1} L^{\dagger}+L \partial_{x}^{-1}=0$. Obviously, $R$ is of the general form

$$
R=a \partial_{x}^{-m}+\text { lower order terms }
$$

and the identity $R^{\dagger}=-R$ implies that if $a$ is not identically zero, then $m$ is odd. Then we have (see [1]):

$$
\begin{gathered}
L^{m} \partial_{x}^{-1}=\left(R \partial_{x}-\partial_{x}^{-1} L^{\dagger} \partial_{x}\right)^{m} \partial_{x}^{-1} \\
=-\left(\partial_{x}^{-1} L^{\dagger} \partial_{x}\right)^{m} \partial_{x}^{-1}+m R\left(\partial_{x}^{-1} L^{\dagger} \partial_{x}\right)^{m-1}+\text { an operator of order less than }-1 \\
=\left(L^{n} \partial_{x}^{-1}\right)^{\dagger}+m a \partial_{x}^{-1}+\text { lower order terms }
\end{gathered}
$$

which contradicts the assumption that $a \neq 0$.

Note that the constraint $(\underline{3.2})$ implies $\left(L^{\dagger}\right)_{+}^{k}=-\left(\partial_{x} L^{k} \partial_{x}^{-1}\right)_{+}=-\left(L^{k}\right)_{+}-\left(\left(\partial_{x} L^{k}\right) \partial_{x}^{-1}\right)_{+}$ which can be rewritten as

$$
B_{k}^{\dagger}=-\partial_{x} B_{k} \partial_{x}^{-1}, \quad k \text { odd }
$$

(taking into account that $b_{k, 0}=0$ ). Using this relation, it is straightforward to check, using the Lax equations, that the constraint (3.2) is indeed invariant under odd flows of the KP hierarchy:

$$
\partial_{t_{k}}\left(L^{\dagger}+\partial_{x} L \partial_{x}^{-1}\right)=0, \quad k \text { odd }
$$

Therefore, the BKP hierarchy is well-defined as a subhierarchy of the KP hierarchy.

The first three differential operators $B_{k}$ are as follows:

$$
\begin{aligned}
& B_{1}=\partial_{x}, \\
& B_{3}=\partial_{x}^{3}+6 u \partial_{x}, \quad u=\frac{1}{2} u_{1}, \\
& B_{5}=\partial_{x}^{5}+10 u \partial_{x}^{3}+10 u^{\prime} \partial_{x}^{2}+v \partial_{x} .
\end{aligned}
$$

An equivalent formulation of the hierarchy is through the zero curvature equations

$$
\partial_{t_{l}} B_{k}-\partial_{t_{k}} B_{l}+\left[B_{k}, B_{l}\right]=0, \quad k, l \text { odd }
$$

The first equation of the BKP hierarchy follows from the zero curvature equation $\partial_{t_{3}} B_{5}-$ $\partial_{t_{5}} B_{3}+\left[B_{5}, B_{3}\right]=0$. The calculations yield the following system of equations for the unknown functions $u, v$ :

$$
\left\{\begin{array}{l}
3 v^{\prime}=10 u_{t_{3}}+20 u^{\prime \prime \prime}+120 u u^{\prime} \\
v_{t_{3}}-6 u_{t_{5}}=v^{\prime \prime \prime}-6 u^{\prime \prime \prime \prime \prime}-60 u u^{\prime \prime \prime}-60 u^{\prime} u^{\prime \prime}+6 u v^{\prime}-6 v u^{\prime},
\end{array}\right.
$$

Note that the variable $v$ can be excluded by passing to the unknown function $U$ such that $U^{\prime}=u$. 


\subsection{The wave function and the tau-function}

The Lax equations and the zero curvature equations are compatibility conditions of the auxiliary linear problems (2.6) for the formal wave function

$$
\psi=\psi\left(x, \mathbf{t}_{\mathrm{o}}, z\right)=W e^{x z+\xi\left(\mathbf{t}_{\mathrm{o}}, z\right)},
$$

where

$$
\xi\left(\mathbf{t}_{\mathrm{o}}, z\right)=\sum_{k \geq 1, \text { odd }} t_{k} z^{k}
$$

As it follows from (2.2), the wave function $\psi=\psi\left(x, \mathbf{t}_{\mathrm{o}}, z\right)$ has the following expansion as $z \rightarrow \infty$ :

$$
\psi\left(x, \mathbf{t}_{\mathrm{o}}, z\right)=e^{x z+\xi\left(\mathbf{t}_{\mathrm{o}}, z\right)}\left(1+\sum_{k \geq 1} \xi_{k} z^{-k}\right) .
$$

As is proved in [3], the wave function satisfies the bilinear relation

$$
\oint_{C_{\infty}} \psi\left(x, \mathbf{t}_{\mathrm{o}}, z\right) \psi\left(x, \mathbf{t}_{\mathrm{o}}^{\prime},-z\right) \frac{d z}{2 \pi i z}=1
$$

valid for all $\mathbf{t}_{\mathrm{o}}, \mathbf{t}_{\mathrm{o}}^{\prime}$. For completeness, we give a sketch of proof here. By virtue of the differential equations (3.4), the bilinear relation is equivalent to vanishing of

$$
b_{m}=\left.\partial_{x^{\prime}}^{m} \oint_{C_{\infty}} \psi\left(x, \mathbf{t}_{\mathrm{o}}, z\right) \psi\left(x^{\prime}, \mathbf{t}_{\mathrm{o}},-z\right) \frac{d z}{2 \pi i z}\right|_{x^{\prime}=x} \quad \text { for all } m \geq 1
$$

with the additional condition that

$$
b_{0}=\oint_{C_{\infty}} \psi\left(x, \mathbf{t}_{\mathrm{o}}, z\right) \psi\left(x, \mathbf{t}_{\mathrm{o}},-z\right) \frac{d z}{2 \pi i z}=1 .
$$

We have:

$$
\begin{gathered}
b_{m}=\left.\oint_{C_{\infty}}\left(\sum_{k \geq 0} \xi_{k}(x) z^{-k}\right) \partial_{x^{\prime}}^{m}\left(\sum_{l \geq 0} \xi_{l}\left(x^{\prime}\right)(-z)^{-l}\right) e^{\left(x-x^{\prime}\right) z} \frac{d z}{2 \pi i z}\right|_{x^{\prime}=x} \\
=\oint_{C_{\infty}}\left(\sum_{k \geq 0} \xi_{k} z^{-k}\right)\left(\partial_{x}-z\right)^{m}\left(\sum_{l \geq 0} \xi_{l}(-z)^{-l}\right) \frac{d z}{2 \pi i z} \\
=\sum_{j+k+l=m}(-1)^{m+j+l}\left(\begin{array}{c}
m \\
j
\end{array}\right) \xi_{k} \partial_{x}^{j} \xi_{l} .
\end{gathered}
$$

But the last expression is the coefficient of $(-1)^{m} \partial_{x}^{-m-1}$ in the operator $W \partial_{x}^{-1} W^{\dagger}$ :

$$
W \partial_{x}^{-1} W^{\dagger}=\partial_{x}^{-1}+\sum_{m \geq 1}(-1)^{m} b_{m} \partial_{x}^{-m-1}
$$

Since $W \partial_{x}^{-1} W^{\dagger}=\partial_{x}^{-1}$, we conclude that $b_{m}=0$ for all $m \geq 1$ and $b_{0}=1$.

The tau-function $\tau=\tau\left(x, \mathbf{t}_{\mathrm{o}}\right)$ of the BKP hierarchy is consistently introduced by the formula

$$
\psi=e^{x z+\xi\left(\mathbf{t}_{\mathrm{o}}, z\right)} \frac{\tau\left(x, \mathbf{t}_{\mathrm{o}}-2\left[z^{-1}\right]_{\mathrm{o}}\right)}{\tau\left(x, \mathbf{t}_{\mathrm{o}}\right)},
$$


where

$$
\mathbf{t}_{\mathrm{o}}+k\left[z^{-1}\right]_{\mathrm{o}} \equiv\left\{t_{1}+\frac{k}{z}, t_{3}+\frac{k}{3 z^{3}}, t_{5}+\frac{k}{5 z^{5}}, \ldots\right\}, \quad k \in \mathbb{Z} .
$$

The proof of the existence of the tau-function is based on the bilinear relation. Let us represent the wave function in the form

$$
\psi\left(x, \mathbf{t}_{\mathrm{o}}, z\right)=e^{x z+\xi\left(\mathbf{t}_{\mathrm{o}}, z\right)} w\left(x, \mathbf{t}_{\mathrm{o}}, z\right)
$$

and set $\mathbf{t}_{\mathrm{o}}^{\prime}=\mathbf{t}_{\mathrm{o}}-2\left[a^{-1}\right]_{\mathrm{o}}$ in the bilinear relation. We have $e^{\xi\left(\mathbf{t}_{\mathrm{o}}-\mathbf{t}_{\mathrm{o}}^{\prime}, z\right)}=\frac{a+z}{a-z}$ and the residue calculus yields

$$
w\left(\mathbf{t}_{\mathrm{o}}, a\right) w\left(\mathbf{t}_{\mathrm{o}}-2\left[a^{-1}\right]_{\mathrm{o}},-a\right)=1,
$$

where we do not indicate the dependence on $x$ for brevity. Next, we set $\mathbf{t}_{\mathrm{o}}^{\prime}=\mathbf{t}_{\mathrm{o}}-$ $2\left[a^{-1}\right]_{\mathrm{o}}-2\left[b^{-1}\right]_{\mathrm{o}}$ in the bilinear relation, so that $e^{\xi\left(\mathbf{t}_{\mathrm{o}}-\mathbf{t}_{\mathrm{o}}^{\prime}, z\right)}=\frac{(a+z)(b+z)}{(a-z)(b-z)}$. In this case the residue calculus yields

$$
w\left(\mathbf{t}_{\mathrm{o}}, a\right) w\left(\mathbf{t}_{\mathrm{o}}-2\left[a^{-1}\right]_{\mathrm{o}}-2\left[b^{-1}\right]_{\mathrm{o}},-a\right)=w\left(\mathbf{t}_{\mathrm{o}}, b\right) w\left(\mathbf{t}_{\mathrm{o}}-2\left[a^{-1}\right]_{\mathrm{o}}-2\left[b^{-1}\right]_{\mathrm{o}},-b\right) .
$$

With the help of (3.16) this latter relation can be rewritten as

$$
\frac{w\left(\mathbf{t}_{\mathrm{o}}, a\right) w\left(\mathbf{t}_{\mathrm{o}}-2\left[a^{-1}\right]_{\mathrm{o}}, b\right)}{w\left(\mathbf{t}_{\mathrm{o}}, b\right) w\left(\mathbf{t}_{\mathrm{o}}-2\left[b^{-1}\right]_{\mathrm{o}}, a\right)}=1 .
$$

Now we are going to show that (3.18) implies that there exists a function $\tau\left(\mathbf{t}_{\mathrm{o}}\right)$ such that

$$
w\left(\mathbf{t}_{\mathrm{o}}, z\right)=\frac{\tau\left(\mathbf{t}_{\mathrm{o}}-2\left[z^{-1}\right]_{\mathrm{o}}\right)}{\tau\left(\mathbf{t}_{\mathrm{o}}\right)} .
$$

To see this, let us represent (3.19) in an equivalent form. Taking logarithm and $z$ derivative, we have from (3.19)

$$
\partial_{z} \log w=2 \sum_{m \geq 1, \text { odd }} z^{-m-1} \partial_{t_{m}} \log \tau\left(\mathbf{t}_{\mathrm{o}}-2\left[z^{-1}\right]_{\mathrm{o}}\right)
$$

or, substituting $\tau\left(\mathbf{t}_{\mathrm{o}}-2\left[z^{-1}\right]_{\mathrm{o}}\right)$ expressed through $w\left(\mathbf{t}_{\mathrm{o}}, z\right)$ and $\tau\left(\mathbf{t}_{\mathrm{o}}\right)$ from (3.19) in the right hand side,

$$
\partial_{z} \log w=2 \partial_{\mathbf{t}_{\mathrm{o}}}(z) \log w+2 \partial_{\mathbf{t}_{\mathrm{o}}}(z) \log \tau,
$$

where $\partial_{\mathbf{t}_{\mathrm{o}}}(z)$ is the differential operator

$$
\partial_{\mathbf{t}_{\mathrm{o}}}(z)=\sum_{j \text { odd }} z^{-j-1} \partial_{t_{j}}
$$

In fact (3.20) is equivalent to (3.19). Indeed, writing (3.20) as $\left(\partial_{z}-2 \partial_{\mathbf{t}_{\mathrm{o}}}(z)\right) \log (w \tau)=0$, we conclude that $w \tau=\rho$ is a function of $\mathbf{t}_{\mathrm{o}}-2\left[z^{-1}\right]_{\mathrm{o}}$, and the normalization condition $w\left(\mathbf{t}_{\mathrm{o}}, \infty\right)=1$ implies that $\rho=\tau$, so we arrive at (3.19).

Equation (3.19) means that

$$
Y_{n}:=\underset{z=\infty}{\operatorname{res}}\left[z^{n}\left(\partial_{z}-2 \partial_{\mathbf{t}_{\mathrm{o}}}(z)\right) \log w\right]=2 \frac{\partial \log \tau}{\partial t_{n}}
$$


where the residue is defined as $\underset{z=\infty}{\operatorname{res}}\left(z^{n-1}\right)=\delta_{n 0}$. Therefore, the existence of the taufunction will be proved if we prove that $\partial_{t_{n}} Y_{m}\left(\mathbf{t}_{\mathrm{o}}\right)=\partial_{t_{m}} Y_{n}\left(\mathbf{t}_{\mathrm{o}}\right)$. Changing $a \rightarrow z, b \rightarrow \zeta$ in (3.18), and applying the operator $\partial_{z}-2 \partial_{\mathbf{t}_{\mathrm{o}}}(z)$ to logarithm of this equality, we rewrite it as

$$
\left(\partial_{z}-2 \partial_{\mathbf{t}_{\mathrm{o}}}(z)\right) \log w\left(\mathbf{t}_{\mathrm{o}}, z\right)-\left(\partial_{z}-2 \partial_{\mathbf{t}_{\mathrm{o}}}(z)\right) \log w\left(\mathbf{t}_{\mathrm{o}}-2\left[\zeta^{-1}\right]_{\mathrm{o}}, z\right)=-2 \partial_{\mathbf{t}_{\mathrm{o}}}(z) \log w_{0}\left(\mathbf{t}_{\mathrm{o}}, \zeta\right)
$$

or

$$
Y_{n}\left(\mathbf{t}_{\mathrm{o}}\right)-Y_{n}\left(\mathbf{t}_{\mathrm{o}}-2\left[\zeta^{-1}\right]_{\mathrm{o}}\right)=-2 \partial_{t_{n}} \log w\left(\mathbf{t}_{\mathrm{o}}, \zeta\right) .
$$

Therefore, denoting $F_{m n}=\partial_{t_{m}} Y_{n}-\partial_{t_{n}} Y_{m}$, we see from (3.21) that

$$
F_{m n}\left(\mathbf{t}_{\mathrm{o}}\right)=F_{m n}\left(\mathbf{t}_{\mathrm{o}}-2\left[\zeta^{-1}\right]_{\mathrm{o}}\right) .
$$

This equality is valid identically in $\zeta$. Expanding its right hand side in a power series,

$$
F_{m n}\left(\mathbf{t}_{\mathrm{o}}-2\left[\zeta^{-1}\right]_{\mathrm{o}}\right)=F_{m n}\left(\mathbf{t}_{\mathrm{o}}\right)-2 \zeta^{-1} \partial_{t_{1}} F_{m n}\left(\mathbf{t}_{\mathrm{o}}\right)-\frac{2}{3} \zeta^{-3}\left(\partial_{t_{3}} F_{m n}\left(\mathbf{t}_{\mathrm{o}}\right)+2 \partial_{t_{1}}^{3} F_{m n}\left(\mathbf{t}_{\mathrm{o}}\right)\right)+\ldots,
$$

we conclude from compating of the $\zeta^{-1}$-terms that $F_{m n}$ does not depend on $t_{1}$. From the $\zeta^{-3}$-terms we see that it does not depend on $t_{3}$ and so on. In this way we can conclude that it does not depend on $t_{k}$ for all (odd) $k$, i.e. $F_{m n}=2 a_{m n}$, where $a_{m n}$ are some constants such that $a_{m n}=-a_{n m}$. Therefore, we can write

$$
Y_{n}=\sum_{m} a_{m n} t_{m}+\partial_{t_{n}} h
$$

with some function $h=h\left(\mathbf{t}_{\mathrm{o}}\right)$. Then from (3.21) we have

$$
-2 \partial_{t_{n}} \log w\left(\mathbf{t}_{\mathrm{o}}, z\right)=\partial_{t_{n}}\left(h\left(\mathbf{t}_{\mathrm{o}}\right)-h\left(\mathbf{t}_{\mathrm{o}}-2\left[z^{-1}\right]_{\mathrm{o}}\right)\right)+2 \sum_{m \text { odd }} \frac{a_{m n}}{m} z^{-m}
$$

or, after integration,

$$
\log w\left(\mathbf{t}_{\mathrm{o}}, z\right)=\frac{1}{2} h\left(\mathbf{t}_{\mathrm{o}}-2\left[z^{-1}\right]_{\mathrm{o}}\right)-\frac{1}{2} h\left(\mathbf{t}_{\mathrm{o}}\right)-\sum_{m \text { odd }} \frac{a_{m n}}{m} z^{-m} t_{n}+\varphi(z),
$$

where $\varphi(z)$ is a function of $z$ only. Substituting this into (3.18), we conclude that $a_{m n}=0$, and so $\partial_{t_{m}} Y_{n}=\partial_{t_{n}} Y_{m}$.

Let us show how to obtain (3.14) up to a common $x$-independent factor in a very easy way. Apply $\partial_{t_{1}}$ to (3.13) and set $\mathbf{t}_{\mathrm{o}}^{\prime}=\mathbf{t}_{\mathrm{o}}-2\left[a^{-1}\right]_{\mathrm{o}}$. The residue calculus yields

$$
\begin{gathered}
2 a\left(w\left(\mathbf{t}_{\mathrm{o}}, a\right) w\left(\mathbf{t}_{\mathrm{o}}-2\left[a^{-1}\right]_{\mathrm{o}},-a\right)-1\right)+2 w^{\prime}\left(\mathbf{t}_{\mathrm{o}}, a\right) w\left(\mathbf{t}_{\mathrm{o}}-2\left[a^{-1}\right]_{\mathrm{o}},-a\right) \\
+\xi_{1}\left(\mathbf{t}_{\mathrm{o}}-2\left[a^{-1}\right]_{\mathrm{o}}\right)-\xi_{1}\left(\mathbf{t}_{\mathrm{o}}\right)=0 .
\end{gathered}
$$

Using (3.16), we conclude from (3.23) that

$$
\partial_{x} \log w\left(\mathbf{t}_{\mathrm{o}}, a\right)=\frac{1}{2}\left(\xi_{1}\left(\mathbf{t}_{\mathrm{o}}\right)-\xi_{1}\left(\mathbf{t}_{\mathrm{o}}-2\left[a^{-1}\right]_{\mathrm{o}}\right)\right) .
$$

Now, setting $\xi_{1}\left(x, \mathbf{t}_{\mathrm{o}}\right)=-2 \partial_{x} \log \tau\left(x, \mathbf{t}_{\mathrm{o}}\right)$ and integrating, we arrive at (3.14) up to a common $x$-independent factor. 
The function $u$ in (3.7) can be also expressed through the tau-function with the help of the following argument. It is a matter of direct verification that the result of the action of the operator $\partial_{x}^{3}+6 u \partial_{x}-\partial_{t_{3}}$ to the wave function $\psi$ of the form (3.12) is $O\left(z^{-1}\right)$ as $z \rightarrow \infty$, i.e.,

$$
\left(\partial_{x}^{3}+6 u \partial_{x}-\partial_{t_{3}}\right) \psi=O\left(z^{-1}\right) e^{x z+\xi\left(\mathbf{t}_{\mathrm{o}}, z\right)}
$$

if the conditions

$$
u=-\frac{1}{2} \xi_{1}^{\prime}, \quad \xi_{1} \xi_{1}^{\prime}-\xi_{1}^{\prime \prime}-\xi_{2}^{\prime}=0
$$

hold true (actually we know that $\left(\partial_{x}^{3}+6 u \partial_{x}-\partial_{t_{3}}\right) \psi=0$ but here we only need the weaker condition (3.25) ). Since from (3.14) it follows that

$$
\xi_{1}=-2 \partial_{x} \log \tau, \quad \xi_{2}=2\left(\partial_{x} \log \tau\right)^{2}+2 \partial_{x}^{2} \log \tau
$$

we have

$$
u=\partial_{x}^{2} \log \tau
$$

and the second equality in (3.26) holds identically.

The change of dependent variables from $u, v$ to the tau-function as in (3.27) and

$$
v=\frac{10}{3} \partial_{t_{3}} \partial_{x} \log \tau+\frac{20}{3} \partial_{x}^{4} \log \tau+20\left(\partial_{x}^{2} \log \tau\right)^{2}
$$

makes the first of the equations (3.9) trivial and the other one turns into the bilinear form [2]

$$
\left(D_{1}^{6}-5 D_{1}^{3} D_{3}-5 D_{3}^{2}+9 D_{1} D_{5}\right) \tau \cdot \tau=0,
$$

where $D_{i}$ are the Hirota operators defined by the rule

$$
\begin{aligned}
& P\left(D_{1}, D_{3}, D_{5}, \ldots\right) \tau \cdot \tau \\
& \quad=\left.P\left(\partial_{y_{1}}, \partial_{y_{3}}, \partial_{y_{5}}, \ldots\right) \tau\left(x, t_{1}+y_{1}, t_{3}+y_{3}, \ldots\right) \tau\left(x, t_{1}-y_{1}, t_{3}-y_{3}, \ldots\right)\right|_{y_{i}=0}
\end{aligned}
$$

for any polynomial $P\left(D_{1}, D_{3}, D_{5}, \ldots\right)$.

As it follows from (3.14), the BKP hierarchy is equivalent to the following relation for the tau-function:

$$
\oint_{C_{\infty}} e^{\xi\left(\mathbf{t}_{\mathrm{o}}-\mathbf{t}_{\mathrm{o}}^{\prime}, z\right)} \tau\left(x, \mathbf{t}_{\mathrm{o}}-2\left[z^{-1}\right]_{\mathrm{o}}\right) \tau\left(x, \mathbf{t}_{\mathrm{o}}^{\prime}+2\left[z^{-1}\right]_{\mathrm{o}}\right) \frac{d z}{2 \pi i z}=\tau\left(x, \mathbf{t}_{\mathrm{o}}\right) \tau\left(x, \mathbf{t}_{\mathrm{o}}^{\prime}\right)
$$

valid for all $\mathbf{t}_{\mathrm{o}}, \mathbf{t}_{\mathrm{o}}^{\prime}$. Set $\mathbf{t}_{\mathrm{o}}^{\prime}=\mathbf{t}_{\mathrm{o}}-2\left[a^{-1}\right]_{\mathrm{o}}-2\left[b^{-1}\right]_{\mathrm{o}}-2\left[c^{-1}\right]_{\mathrm{o}}$, then

$$
e^{\xi\left(\mathbf{t}_{\mathrm{o}}-\mathbf{t}_{\mathrm{o}}^{\prime}, z\right)}=\frac{(a+z)(b+z)(c+z)}{(a-z)(b-z)(c-z)}
$$

and the residue calculus in (3.30) gives the following equation:

$$
\begin{gathered}
(a+b)(a+c)(b-c) \tau^{[a]} \tau^{[b c]}+(b+a)(b+c)(c-a) \tau^{[b]} \tau^{[a c]} \\
+(c+a)(c+b)(a-b) \tau^{[c]} \tau^{[a b]}+(a-b)(b-c)(c-a) \tau \tau^{[a b c]}=0,
\end{gathered}
$$


where $\tau^{[a]}=\tau\left(x, \mathbf{t}_{\mathrm{o}}+2\left[a^{-1}\right]_{\mathrm{o}}\right), \tau^{[a b]}=\tau\left(x, \mathbf{t}_{\mathrm{o}}+2\left[a^{-1}\right]_{\mathrm{o}}+2\left[b^{-1}\right]_{\mathrm{o}}\right)$, and so on. Equation (3.31) should be valid for all $a, b, c$. Taking the limit $c \rightarrow \infty$, we get the equation

$$
\tau \tau^{[a b]}\left(1+\frac{1}{a+b} \partial_{t_{1}} \log \frac{\tau}{\tau^{[a b]}}\right)=\tau^{[a]} \tau^{[b]}\left(1+\frac{1}{a-b} \partial_{t_{1}} \log \frac{\tau^{[b]}}{\tau^{[a]}}\right)
$$

This is the equation for the tau-function of the BKP hierarchy. It should hold for all

$a, b$. The differential equations of the hierarchy are obtained by expanding it in inverse powers of $a, b$.

\subsection{The BKP hierarchy and the KP hierarchy}

Here we compare the spaces of solutions to the BKP and KP hierarchies by an explicit embedding of the former into the latter on the level of tau-functions.

We begin with the adjoint wave function:

$$
\psi^{\dagger}\left(x, \mathbf{t}_{\mathrm{o}}, z\right)=\left(W^{\dagger}\right)^{-1} e^{-x z-\xi\left(\mathbf{t}_{\mathrm{o}}, z\right)}=\partial_{x} W \partial_{x}^{-1} e^{-x z-\xi\left(\mathbf{t}_{\mathrm{o}}, z\right)}=-z^{-1} \partial_{x} \psi\left(x, \mathbf{t}_{\mathrm{o}},-z\right),
$$

so that the bilinear relation (2.13) reads

$$
\oint_{C_{\infty}} \psi\left(x, \mathbf{t}_{\mathrm{o}}, z\right) \partial_{x} \psi\left(x, \mathbf{t}_{\mathrm{o}}^{\prime},-z\right) \frac{d z}{2 \pi i z}=0
$$

which is a consequence of (3.13). Using (2.11), (2.12), one can write (3.33) in terms of the KP tau-function:

$$
e^{-x z} \frac{\tau^{\mathrm{KP}}\left(x, \dot{\mathbf{t}}+\left[z^{-1}\right]\right)}{\tau^{\mathrm{KP}}(x, \dot{\mathbf{t}})}=-z^{-1} \partial_{x}\left(\frac{e^{-x z} \tau^{\mathrm{KP}}\left(x, \dot{\mathbf{t}}-\left[-z^{-1}\right]\right)}{\tau^{\mathrm{KP}}(x, \dot{\mathbf{t}})}\right)
$$

or

$$
\partial_{x} \log \frac{\tau^{\mathrm{KP}}\left(x, \dot{\mathbf{t}}-\left[z^{-1}\right]\right)}{\tau^{\mathrm{KP}}(x, \dot{\mathbf{t}})}=-z\left(1-\frac{\tau^{\mathrm{KP}}\left(x, \dot{\mathbf{t}}+\left[-z^{-1}\right]\right)}{\tau^{\mathrm{KP}}\left(x, \dot{\mathbf{t}}-\left[z^{-1}\right]\right)}\right)
$$

where we use the short-hand notation $\dot{\mathbf{t}}=\left\{t_{1}, 0, t_{3}, 0, \ldots\right\}$. Shifting the times $\mathbf{t}_{\mathrm{o}}$, we can rewrite this as

$$
\partial_{x} \log \frac{\tau^{\mathrm{KP}}\left(t_{1},-\frac{1}{2} z^{-2}, t_{3},-\frac{1}{4} z^{-4}, \ldots\right)}{\tau^{\mathrm{KP}}\left(t_{1}+z^{-1}, 0, t_{3}+\frac{1}{3} z^{-3}, 0, \ldots\right)}=-z+z \frac{\tau^{\mathrm{KP}}\left(t_{1}, \frac{1}{2} z^{-2}, t_{3}, \frac{1}{4} z^{-4}, \ldots\right)}{\tau^{\mathrm{KP}}\left(t_{1},-\frac{1}{2} z^{-2}, t_{3},-\frac{1}{4} z^{-4}, \ldots\right)},
$$

or, subtracting these equalities with $z$ and $-z$,

$$
\partial_{x} \log \frac{\tau^{\mathrm{KP}}\left(x, t_{1}-z^{-1}, 0, t_{3}-\frac{1}{3} z^{-3}, 0, \ldots\right)}{\tau^{\mathrm{KP}}\left(x, t_{1}+z^{-1}, 0, t_{3}+\frac{1}{3} z^{-3}, 0, \ldots\right)}=-2 z+2 z \frac{\tau^{\mathrm{KP}}\left(x, t_{1}, \frac{1}{2} z^{-2}, t_{3}, \frac{1}{4} z^{-4}, \ldots\right)}{\tau^{\mathrm{KP}}\left(x, t_{1},-\frac{1}{2} z^{-2}, t_{3},-\frac{1}{4} z^{-4}, \ldots\right)} .
$$

Comparing this with the KP hierarchy in the form (2.16) at $z_{2}=-z_{1}=z$, we conclude that

$$
\left(\tau^{\mathrm{KP}}\left(x, \dot{\mathbf{t}}-\left[z^{-1}\right]\right)\right)^{2}=\tau^{\mathrm{KP}}(x, \dot{\mathbf{t}}) \tau^{\mathrm{KP}}\left(x, \dot{\mathbf{t}}-2\left[z^{-1}\right]_{\mathrm{o}}\right)
$$

(in the second tau-function in the right hand side, the even times are equal to 0 ). This is the constraint which distinguishes solutions to the BKP hierarchy among all solutions to the KP hierarchy. 
Another way to come to (3.37) is to notice that we have two different expressions for the wave function $\psi$ (one in terms of the KP tau-function $\tau^{\mathrm{KP}}$ and the other in terms of the BKP tau-function $\tau$ ) from which it follows that

$$
\frac{\tau^{\mathrm{KP}}\left(x, \dot{\mathbf{t}}-\left[z^{-1}\right]\right)}{\tau^{\mathrm{KP}}(x, \dot{\mathbf{t}})}=\frac{\tau\left(x, \mathbf{t}_{\mathrm{o}}-2\left[z^{-1}\right]_{\mathrm{o}}\right)}{\tau\left(x, \mathbf{t}_{\mathrm{o}}\right)}
$$

or, after a shift of the times $\mathbf{t}_{\mathrm{o}}$,

$$
\log \frac{\tau^{\mathrm{KP}}\left(x, t_{1},-\frac{1}{2} z^{-2}, t_{3},-\frac{1}{4} z^{-4}, \ldots\right)}{\tau^{\mathrm{KP}}\left(x, t_{1}+z^{-1}, 0, t_{3}+\frac{1}{3} z^{-3}, 0, \ldots\right)}=\log \frac{\tau\left(x, \mathbf{t}_{\mathrm{o}}-\left[z^{-1}\right]_{\mathrm{o}}\right)}{\tau\left(x, \mathbf{t}_{\mathrm{o}}-\left[-z^{-1}\right]_{\mathrm{o}}\right)} .
$$

The right hand side is and odd function of $z$, therefore, we have

$$
\log \frac{\tau^{\mathrm{KP}}\left(x, t_{1},-\frac{1}{2} z^{-2}, t_{3},-\frac{1}{4} z^{-4}, \ldots\right)}{\tau^{\mathrm{KP}}\left(x, t_{1}+z^{-1}, 0, t_{3}+\frac{1}{3} z^{-3}, 0, \ldots\right)}+\log \frac{\tau^{\mathrm{KP}}\left(x, t_{1},-\frac{1}{2} z^{-2}, t_{3},-\frac{1}{4} z^{-4}, \ldots\right)}{\tau^{\mathrm{KP}}\left(x, t_{1}-z^{-1}, 0, t_{3}-\frac{1}{3} z^{-3}, 0, \ldots\right)}=0
$$

which is (3.37).

The constraint (3.37) should be valid for all values of $t_{1}, t_{3}, \ldots$ and $z$. Expanding it in powers of $z$, one can represent it as an infinite number of differential constraints the first of which is

$$
\left.\left(\partial_{t_{2}}+\partial_{t_{1}}^{2}\right) \log \tau^{\mathrm{KP}}\right|_{t_{2 k}=0}=0, \quad k \geq 1
$$

This constraint was mentioned in [14].

Let us represent (3.37) in the form

$$
\frac{\tau^{\mathrm{KP}}\left(x, \dot{\mathbf{t}}-2\left[z^{-1}\right]_{\mathrm{o}}\right)}{\tau^{\mathrm{KP}}(x, \dot{\mathbf{t}})}=\left(\frac{\tau^{\mathrm{KP}}\left(x, \dot{\mathbf{t}}-\left[z^{-1}\right]\right)}{\tau^{\mathrm{KP}}(x, \dot{\mathbf{t}})}\right)^{2}=\frac{\tau^{2}\left(x, \mathbf{t}_{\mathrm{o}}-2\left[z^{-1}\right]_{\mathrm{o}}\right)}{\tau^{2}\left(x, \mathbf{t}_{\mathrm{o}}\right)} .
$$

It follows from here that

$$
\tau\left(x, \mathbf{t}_{\mathrm{o}}\right)=C \sqrt{\tau^{\mathrm{KP}}(x, \dot{\mathbf{t}})}
$$

and that $\tau^{\mathrm{KP}}(x, \dot{\mathbf{t}})$ is a full square, i.e., $\sqrt{\tau^{\mathrm{KP}}(x, \dot{\mathbf{t}})}$ does not have square root singularities in all the times.

\subsection{Examples: soliton solutions}

$N$-soliton solutions of the BKP hierarchy are obtained by imposing certain constraints on the parameters of $2 N$-soliton solutions to the KP hierarchy. The tau-function of the BKP hierarchy is related to the KP tau-function as $\tau=\sqrt{\tau^{\mathrm{KP}}}$, with "even" times $t_{2 k}$ put equal to zero and it is implied that the parameters of the KP tau-function $\tau^{\mathrm{KP}}$ are chosen in a special way. With this choice, $\tau^{\mathrm{KP}}$ is a full square, i.e., $\tau$ does not have square root singularities.

\subsubsection{One-soliton solution}

The tau-function for one BKP soliton is the square root of a specialization of 2-soliton tau-function of the KP hierarchy:

$$
\left.\tau^{\mathrm{KP}}\right|_{t_{2 k}=0}=1+\alpha(p-q) w+\frac{\alpha^{2}}{4}(p-q)^{2} w^{2}=\left(1+\frac{\alpha}{2}(p-q) w\right)^{2}
$$


where

$$
w=e^{(p+q) x+\xi\left(\mathbf{t}_{\mathrm{o}}, p\right)+\xi\left(\mathbf{t}_{\mathrm{o}}, q\right)}, \quad \xi\left(\mathbf{t}_{\mathrm{o}}, z\right) \text { is given by }(3.11)
$$

and $\alpha, p, q$ are arbitrary parameters. Therefore, the tau-function of the BKP hierarchy is

$$
\tau=1+\frac{\alpha}{2}(p-q) w
$$

It is an entire function of $x$.

Note that the extension of the tau-function $\tau^{\mathrm{KP}}$ to the modified KP hierarchy $(\mathrm{mKP})$ reads

$$
\tau_{n}^{\mathrm{mKP}}=1+\alpha\left(-q(-p / q)^{n}+p(-q / p)^{n}\right) w+\frac{\alpha^{2}}{4}(p-q)^{2} w^{2},
$$

where $n$ is the integer-valued "zeroth time" (clearly, $\tau^{\mathrm{KP}}=\tau_{0}^{\mathrm{mKP}}=\tau_{1}^{\mathrm{mKP}}$ ). Then we see that the parameters of the soliton solutions are such that $\tau_{1-n}^{\mathrm{mKP}}=\tau_{n}^{\mathrm{mKP}}$, which is the constraint necessary for the BKP hierarchy [4, 21].

The bilinear identity (3.13), which in the present case has the explicit form

$$
\begin{gathered}
\oint_{C_{\infty}} e^{\xi\left(\mathbf{t}_{\mathrm{o}}, z\right)-\xi\left(\mathbf{t}_{\mathrm{o}}^{\prime}, z\right)}\left(1+\frac{\alpha}{2} \frac{(z-p)(z-q)}{(z+p)(z+q)}(p-q) w\right)\left(1+\frac{\alpha}{2} \frac{(z+p)(z+q)}{(z-p)(z-q)}(p-q) w^{\prime}\right) \frac{d z}{2 \pi i z} \\
=\left(1+\frac{\alpha}{2}(p-q) w\right)\left(1+\frac{\alpha}{2}(p-q) w^{\prime}\right)
\end{gathered}
$$

can be checked directly by the residue calculus. (Here $w^{\prime}=e^{(p+q) x+\xi\left(\mathbf{t}_{\mathrm{o}}^{\prime}, p\right)+\xi\left(\mathbf{t}_{\mathrm{o}}^{\prime}, q\right)}$.)

\subsubsection{Multi-soliton solutions}

The general KP tau-function for $2 N$-soliton solution has $6 N$ arbitrary parameters $\alpha_{i}, p_{i}$, $q_{i}(i=1, \ldots, 2 N)$ and is given by

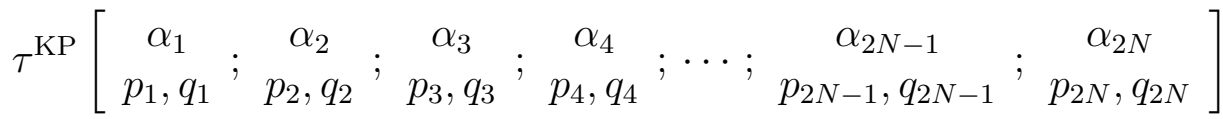

$$
\begin{aligned}
& =\operatorname{det}_{1 \leq i, k \leq 2 N}\left(\delta_{i k}+\alpha_{i} \frac{p_{i}-q_{i}}{p_{i}-q_{k}} e^{\left(p_{i}-q_{i}\right) x+\xi\left(\mathbf{t}, p_{i}\right)-\xi\left(\mathbf{t}, q_{i}\right)}\right) .
\end{aligned}
$$

The $N$-soliton tau-function of the BKP hierarchy is the square root of the $\tau^{\mathrm{KP}}$ specialized as

$$
\tau^{\mathrm{KP}}\left[\begin{array}{ccc}
-q_{1} \alpha_{1} \\
p_{1},-q_{1}
\end{array} ; \begin{array}{c}
p_{1} \alpha_{1},-p_{1} \\
q_{1}, p_{2} \alpha_{2} \\
p_{2},-q_{2}
\end{array} ; \begin{array}{c}
p_{2} \alpha_{2} \\
q_{2},-p_{2}
\end{array} ; \cdots ; \begin{array}{c}
-q_{N} \alpha_{N} \\
p_{N},-q_{N}
\end{array} ; \begin{array}{c}
p_{N} \alpha_{N} \\
q_{N},-p_{N}
\end{array}\right],
$$

where it is implied that even times evolution is frozen $\left(t_{2 k}=0\right.$ for all $\left.k \geq 1\right)$. It can be proved that this KP tau-function is a full square, i.e., $\tau=\sqrt{\tau^{\mathrm{KP}}}$ is an entire function of the times (no square root singularities!).

\section{The CKP hierarchy}

In this section we present the main formulas related to the CKP hierarchy with some details. The main references are [3, 7, 8]. 


\subsection{The CKP equation and the hierarchy}

The set of independent variables ("times") is $\mathbf{t}_{\mathrm{o}}=\left\{t_{1}, t_{3}, t_{5}, \ldots\right\}$. Like in the BKP hierarchy, they are indexed by positive odd numbers. We set $t_{1}=x+$ const. The main object is the $L$-operator (2.1) with the constraint

$$
L^{\dagger}=-L
$$

It is instructive to reformulate this constraint in terms of the wave operator $W$ (2.2) such that $L=W \partial_{x} W^{-1}$. The constraint implies that $W^{\dagger} W$ commutes with $\partial_{x}$, i.e., it is a pseudo-differential operator with constant coefficients. The freedom in the definition of the wave operator can be fixed by demanding that $W^{\dagger} W=1$, i.e.

$$
W^{\dagger}=W^{-1} .
$$

The evolution equations (the Lax equations) and the zero curvature equations have the same form (3.4) and (3.8) as in the BKP hierarchy. By applying the $\dagger$-operation to the evolution equations (3.4) it is not difficult to see that they are consistent with the constraint (4.1), i.e., $\partial_{t_{k}}\left(L+L^{\dagger}\right)=0$ for odd $k$, so the CKP hierarchy is well-defined.

Clearly, the differential operators $B_{k}$ satisfy $B_{k}^{\dagger}=-B_{k}$ (for odd $k$ ). In particular,

$$
\begin{aligned}
& B_{1}=\partial_{x}, \\
& B_{3}=\partial_{x}^{3}+6 u \partial_{x}+3 u^{\prime}, \\
& B_{5}=\partial_{x}^{5}+10 u \partial_{x}^{3}+15 u^{\prime} \partial_{x}^{2}+v \partial_{x}+\frac{1}{2}\left(v^{\prime}-5 u^{\prime \prime \prime}\right) .
\end{aligned}
$$

where $u^{\prime} \equiv \partial_{x} u, u=\frac{1}{2} u_{1}$. Since $B_{1}=\partial_{x}$, it follows from (3.4), like in the KP hierarchy, that the evolution in the time $t_{1}$ is simply the shift of $x$, i.e. the solutions depend on $x+t_{1}$.

The first equation of the CKP hierarchy follows from the zero curvature equation $\partial_{t_{3}} B_{5}-\partial_{t_{5}} B_{3}+\left[B_{5}, B_{3}\right]=0$ with $B_{3}, B_{5}$ as in (4.3). The calculations yield the following system of equations for the unknown functions $u, v$ :

$$
\left\{\begin{array}{l}
10 \partial_{t_{3}} u=3 v^{\prime}-35 u^{\prime \prime \prime}-120 u u^{\prime} \\
6 \partial_{t_{5}} u-\partial_{t_{3}} v=\frac{57}{2} u^{\prime \prime \prime \prime \prime}+150 u u^{\prime \prime \prime}+180 u^{\prime} u^{\prime \prime}-\frac{5}{2} v^{\prime \prime \prime}+6 v u^{\prime}-6 u v^{\prime} .
\end{array}\right.
$$

Note that the variable $v$ can be excluded by passing to the unknown function $U$ such that $U^{\prime}=u$.

\subsection{The wave function and the tau-function}

Like in the KP and BKP hierarchies, the Lax equations and the zero curvature equations are compatibility conditions of the auxiliary linear problems

$$
\partial_{t_{k}} \Psi=B_{k} \Psi, \quad L \Psi=z \Psi
$$


for the formal wave function

$$
\Psi=\Psi\left(x, \mathbf{t}_{\mathrm{o}}, z\right)=W e^{x z+\xi\left(\mathbf{t}_{\mathrm{o}}, z\right)},
$$

where $z$ is the spectral parameter and $\xi\left(\mathbf{t}_{\mathrm{o}}, z\right)$ is defined in (3.11). The wave function has the following expansion as $z \rightarrow \infty$ :

$$
\Psi\left(x, \mathbf{t}_{\mathrm{o}}, z\right)=e^{x z+\xi\left(\mathbf{t}_{\mathrm{o}}, z\right)}\left(1+\sum_{k \geq 1} \xi_{k} z^{-k}\right) .
$$

As is proved in [3], it satisfies the bilinear relation

$$
\oint_{C_{\infty}} \Psi\left(x, \mathbf{t}_{\mathrm{o}}, z\right) \Psi\left(x, \mathbf{t}_{\mathrm{o}}^{\prime},-z\right) \frac{d z}{2 \pi i}=0
$$

valid for all $\mathbf{t}_{\mathrm{o}}, \mathbf{t}_{\mathrm{o}}^{\prime}$. Here is a sketch of proof. By virtue of the differential equations (4.5), the bilinear relation is equivalent to vanishing of

$$
b_{m}=\left.\partial_{x^{\prime}}^{m} \oint_{C_{\infty}} \Psi\left(x, \mathbf{t}_{\mathrm{o}}, z\right) \Psi\left(x^{\prime}, \mathbf{t}_{\mathrm{o}},-z\right) \frac{d z}{2 \pi i}\right|_{x^{\prime}=x} \quad \text { for all } m \geq 0 .
$$

The further calculation is similar to the one done in the BKP case. We have:

$$
\begin{gathered}
b_{m}=\left.\oint_{C_{\infty}}\left(\sum_{k \geq 0} \xi_{k}(x) z^{-k}\right) \partial_{x^{\prime}}^{m}\left(\sum_{l \geq 0} \xi_{l}\left(x^{\prime}\right)(-z)^{-l}\right) e^{\left(x-x^{\prime}\right) z} \frac{d z}{2 \pi i}\right|_{x^{\prime}=x} \\
=\oint_{C_{\infty}}\left(\sum_{k \geq 0} \xi_{k} z^{-k}\right)\left(\partial_{x}-z\right)^{m}\left(\sum_{l \geq 0} \xi_{l}(-z)^{-l}\right) \frac{d z}{2 \pi i} \\
=\sum_{j+k+l=m+1}(-1)^{m+j+l}\left(\begin{array}{c}
m \\
j
\end{array}\right) \xi_{k} \partial_{x}^{j} \xi_{l} .
\end{gathered}
$$

It remains to notice that this expression is the coefficient of $(-1)^{m} \partial_{x}^{-m-1}$ in the operator $W W^{\dagger}: W W^{\dagger}=1+\sum_{m \geq 0}(-1)^{m} b_{m} \partial_{x}^{-m-1}$. Since $W W^{\dagger}=1$ (see (4.2) $)$, we conclude that $b_{m}=0$ for all $m \geq 0$.

The tau-function $\tau=\tau\left(x, \mathbf{t}_{\mathrm{o}}\right)$ of the CKP hierarchy is consistently introduced by the formula [7, 8]

$$
\Psi=e^{x z+\xi\left(\mathbf{t}_{\mathrm{o}}, z\right)} G\left(x, \mathbf{t}_{\mathrm{o}}, z\right) \frac{\tau\left(x, \mathbf{t}_{\mathrm{o}}-2\left[z^{-1}\right]_{\mathrm{o}}\right)}{\tau\left(x, \mathbf{t}_{\mathrm{o}}\right)},
$$

where

$$
G\left(x, \mathbf{t}_{\mathrm{o}}, z\right)=\left(1+z^{-1} \partial_{t_{1}} \log \frac{\tau\left(x, \mathbf{t}_{\mathrm{o}}-2\left[z^{-1}\right]_{\mathrm{o}}\right)}{\tau\left(x, \mathbf{t}_{\mathrm{o}}\right)}\right)^{1 / 2}
$$

(cf. (3.14), where there is no factor $G\left(x, \mathbf{t}_{\mathrm{o}}, z\right)$ ). The formula (4.9) (and the very existence of the tau-function) is based on the bilinear relation (4.8) The proof can be found in [11. For completeness, we give it here. Let us represent the wave function in the form

$$
\Psi\left(x, \mathbf{t}_{\mathrm{o}}, z\right)=e^{x z+\xi\left(\mathbf{t}_{\mathrm{o}}, z\right)} w\left(x, \mathbf{t}_{\mathrm{o}}, z\right)
$$


and set $\mathbf{t}_{\mathrm{o}}^{\prime}=\mathbf{t}_{\mathrm{o}}-2\left[a^{-1}\right]_{\mathrm{o}}$ in the bilinear relation. We have $e^{\xi\left(\mathbf{t}_{\mathrm{o}}-\mathbf{t}_{\mathrm{o}}^{\prime}, z\right)}=\frac{a+z}{a-z}$. The residue calculus in (4.8) yields

$$
w\left(\mathbf{t}_{\mathrm{o}}, a\right) w\left(\mathbf{t}_{\mathrm{o}}-2\left[a^{-1}\right]_{\mathrm{o}},-a\right)=f(\mathbf{t}, a)
$$

where

$$
f\left(\mathbf{t}_{\mathrm{o}}, z\right)=1+\frac{1}{2 z}\left(\xi_{1}\left(\mathbf{t}_{\mathrm{o}}\right)-\xi_{1}\left(\mathbf{t}_{\mathrm{o}}-2\left[z^{-1}\right]_{\mathrm{o}}\right)\right)
$$

(here and below we do not indicate the $x$-dependence for brevity). Next, we set $\mathbf{t}_{\mathrm{o}}^{\prime}=$ $\mathbf{t}_{\mathrm{o}}-2\left[a^{-1}\right]_{\mathrm{o}}-2\left[b^{-1}\right]_{\mathrm{o}}$ in the bilinear relation and the residue calculus yields

$$
\begin{gathered}
\frac{a+b}{a-b}\left(a w\left(\mathbf{t}_{\mathrm{o}}, a\right) w\left(\mathbf{t}_{\mathrm{o}}-2\left[a^{-1}\right]_{\mathrm{o}}-2\left[b^{-1}\right]_{\mathrm{o}},-a\right)-b w\left(\mathbf{t}_{\mathrm{o}}, b\right) w\left(\mathbf{t}_{\mathrm{o}}-2\left[a^{-1}\right]_{\mathrm{o}}-2\left[b^{-1}\right]_{\mathrm{o}},-b\right)\right) \\
=a+b+\frac{1}{2}\left(\xi_{1}\left(\mathbf{t}_{\mathrm{o}}\right)-\xi_{1}\left(\mathbf{t}_{\mathrm{o}}-2\left[a^{-1}\right]_{\mathrm{o}}-2\left[b^{-1}\right]_{\mathrm{o}}\right) .\right.
\end{gathered}
$$

Expressing $w(\ldots,-a), w(\ldots,-b)$ through $w(\ldots, a), w(\ldots, b)$ by means of the relation (4.11), we can represent this equation in the form

$$
\begin{gathered}
\frac{1}{a-b}\left(a f\left(\mathbf{t}_{\mathrm{o}}-2\left[b^{-1}\right]_{\mathrm{o}}, a\right) \frac{w\left(\mathbf{t}_{\mathrm{o}}, a\right)}{w\left(\mathbf{t}_{\mathrm{o}}-2\left[b^{-1}\right]_{\mathrm{o}}, a\right)}-b f\left(\mathbf{t}_{\mathrm{o}}-2\left[a^{-1}\right]_{\mathrm{o}}, b\right) \frac{w\left(\mathbf{t}_{\mathrm{o}}, b\right)}{w\left(\mathbf{t}_{\mathrm{o}}-2\left[a^{-1}\right]_{\mathrm{o}}, b\right)}\right) \\
=1+\frac{\xi_{1}\left(\mathbf{t}_{\mathrm{o}}\right)-\xi_{1}\left(\mathbf{t}_{\mathrm{o}}-2\left[a^{-1}\right]_{\mathrm{o}}-2\left[b^{-1}\right]_{\mathrm{o}}\right)}{2(a+b)} .
\end{gathered}
$$

Shifting here $\mathbf{t}_{\mathrm{o}} \rightarrow \mathbf{t}_{\mathrm{o}}+2\left[b^{-1}\right]_{\mathrm{o}}$, changing the sign of $b$ (i.e, substituting $b \rightarrow-b$ ) and using (4.11) again after that (in the second term in the left hand side), we arrive at the equivalent equation

$$
\begin{gathered}
\frac{1}{a+b}\left(a f\left(\mathbf{t}_{\mathrm{o}}, a\right) \frac{w\left(\mathbf{t}_{\mathrm{o}}-2\left[b^{-1}\right]_{\mathrm{o}}, a\right)}{w\left(\mathbf{t}_{\mathrm{o}}, a\right)}-b f\left(\mathbf{t}_{\mathrm{o}}, b\right) \frac{w\left(\mathbf{t}_{\mathrm{o}}-2\left[a^{-1}\right]_{\mathrm{o}}, b\right)}{w\left(\mathbf{t}_{\mathrm{o}}, b\right)}\right) \\
=1+\frac{\xi_{1}\left(\mathbf{t}_{\mathrm{o}}-2\left[b^{-1}\right]_{\mathrm{o}}\right)-\xi_{1}\left(\mathbf{t}_{\mathrm{o}}-2\left[a^{-1}\right]_{\mathrm{o}}\right)}{2(a-b)} .
\end{gathered}
$$

Together equations (4.14), (4.15) form the system of two equations

$$
\left\{\begin{array}{l}
\frac{1}{a-b}\left(a f\left(\mathbf{t}_{\mathrm{o}}-2\left[b^{-1}\right]_{\mathrm{o}}, a\right) X^{-1}-b f\left(\mathbf{t}_{\mathrm{o}}-2\left[a^{-1}\right]_{\mathrm{o}}, b\right) Y^{-1}\right)=\frac{a f\left(\mathbf{t}_{\mathrm{o}}, a\right)+b f\left(\mathbf{t}_{\mathrm{o}}-2\left[a^{-1}\right]_{\mathrm{o}}, b\right)}{a+b} \\
\frac{1}{a+b}\left(a f\left(\mathbf{t}_{\mathrm{o}}, a\right) X-b f\left(\mathbf{t}_{\mathrm{o}}, b\right) Y\right)=\frac{a f\left(\mathbf{t}_{\mathrm{o}}, a\right)-b f\left(\mathbf{t}_{\mathrm{o}}, b\right)}{a-b}
\end{array}\right.
$$

for the two "unknown quantities"

$$
X=\frac{w\left(\mathbf{t}_{\mathrm{o}}-2\left[b^{-1}\right]_{\mathrm{o}}, a\right)}{w\left(\mathbf{t}_{\mathrm{o}}, a\right)}, \quad Y=\frac{w\left(\mathbf{t}_{\mathrm{o}}-2\left[a^{-1}\right]_{\mathrm{o}}, b\right)}{w\left(\mathbf{t}_{\mathrm{o}}, b\right)} .
$$


The next step is to multiply the two equations (4.16). After some algebra, one obtains the following simple relation:

$$
\frac{Y}{X}=\frac{w\left(\mathbf{t}_{\mathrm{o}}, a\right) w\left(\mathbf{t}_{\mathrm{o}}-2\left[a^{-1}\right]_{\mathrm{o}}, b\right)}{w\left(\mathbf{t}_{\mathrm{o}}, b\right) w\left(\mathbf{t}_{\mathrm{o}}-2\left[b^{-1}\right]_{\mathrm{o}}, a\right)}=\left(\frac{f\left(\mathbf{t}_{\mathrm{o}}, a\right) f\left(\mathbf{t}_{\mathrm{o}}-2\left[a^{-1}\right]_{\mathrm{o}}, b\right)}{f\left(\mathbf{t}_{\mathrm{o}}, b\right) f\left(\mathbf{t}_{\mathrm{o}}-2\left[b^{-1}\right]_{\mathrm{o}}, a\right)}\right)^{1 / 2} .
$$

In the calculations, the identity

$$
a f\left(\mathbf{t}_{\mathrm{o}}, a\right)-a f\left(\mathbf{t}_{\mathrm{o}}-2\left[b^{-1}\right]_{\mathrm{o}}, a\right)-b f\left(\mathbf{t}_{\mathrm{o}}, b\right)+b f\left(\mathbf{t}_{\mathrm{o}}-2\left[a^{-1}\right]_{\mathrm{o}}, b\right)=0
$$

has been used. Introducing $w_{0}\left(\mathbf{t}_{\mathrm{o}}, z\right)=w\left(\mathbf{t}_{\mathrm{o}}, z\right) f^{-1 / 2}\left(\mathbf{t}_{\mathrm{o}}, z\right)$, we get from it the relation

$$
\frac{w_{0}\left(\mathbf{t}_{\mathrm{o}}, a\right) w_{0}\left(\mathbf{t}_{\mathrm{o}}-2\left[a^{-1}\right]_{\mathrm{o}}, b\right)}{w_{0}\left(\mathbf{t}_{\mathrm{o}}, b\right) w_{0}\left(\mathbf{t}_{\mathrm{o}}-2\left[b^{-1}\right]_{\mathrm{o}}, a\right)}=1
$$

which has the same form as (3.18) for the BKP hierarchy, with the change in the notation $w \rightarrow w_{0}$.

As soon as the relation of the form (4.20) is established, the rest of the argument is the same as for the BKP hierarchy. In the same way as in section 3.2 we can prove that there exists a function $\tau\left(\mathbf{t}_{\mathrm{o}}\right)$ such that

$$
w_{0}\left(\mathbf{t}_{\mathrm{o}}, z\right)=\frac{\tau\left(\mathbf{t}_{\mathrm{o}}-2\left[z^{-1}\right]_{\mathrm{o}}\right)}{\tau\left(\mathbf{t}_{\mathrm{o}}\right)} .
$$

This function is called the tau-function of the CKP hierarchy. Finally, writing $w\left(\mathbf{t}_{\mathrm{o}}, z\right)=$ $f^{1 / 2}\left(\mathbf{t}_{\mathrm{o}}, z\right) w_{0}\left(\mathbf{t}_{\mathrm{o}}, z\right)$ and noting that $f\left(\mathbf{t}_{\mathrm{o}}, z\right)=1+O\left(z^{-2}\right)$, we see that

$$
\xi_{1}\left(\mathbf{t}_{\mathrm{o}}\right)=-2 \partial_{t_{1}} \log \tau\left(\mathbf{t}_{\mathrm{o}}\right)
$$

and, recalling (4.12), we arrive at (4.9) with $G=f^{1 / 2}$.

Let us show that equation (4.9) can be obtained up to a common $x$-independent factor in the following easy way [7, 8]. Apply $\partial_{t_{1}}$ to (4.8) and set $\mathbf{t}_{\mathrm{o}}^{\prime}=\mathbf{t}_{\mathrm{o}}-2\left[a^{-1}\right]_{\mathrm{o}}$. The residue calculus yields

$$
\begin{gathered}
2 a^{2}\left(1-w\left(\mathbf{t}_{\mathrm{o}}, a\right) w\left(\mathbf{t}_{\mathrm{o}}-2\left[a^{-1}\right]_{\mathrm{o}},-a\right)\right)-2 a w^{\prime}\left(\mathbf{t}_{\mathrm{o}}, a\right) w\left(\mathbf{t}_{\mathrm{o}}-2\left[a^{-1}\right]_{\mathrm{o}},-a\right) \\
+2 a\left(\xi_{1}\left(\mathbf{t}_{\mathrm{o}}\right)-\xi_{1}\left(\mathbf{t}_{\mathrm{o}}-2\left[a^{-1}\right]_{\mathrm{o}}\right)\right)+\xi_{2}\left(\mathbf{t}_{\mathrm{o}}-2\left[a^{-1}\right]_{\mathrm{o}}\right)+\xi_{2}\left(\mathbf{t}_{\mathrm{o}}\right)+\xi_{1}^{\prime}\left(\mathbf{t}_{\mathrm{o}}\right) \\
-\xi_{1}\left(\mathbf{t}_{\mathrm{o}}\right) \xi_{1}\left(\mathbf{t}_{\mathrm{o}}-2\left[a^{-1}\right]_{\mathrm{o}}\right)=0
\end{gathered}
$$

where prime means the $x$-derivative and we again do not indicate the dependence on $x$ explicitly. Tending $a \rightarrow \infty$ in (4.23), we get the relation

$$
2 \xi_{2}\left(\mathbf{t}_{\mathrm{o}}\right)=\xi_{1}^{2}\left(\mathbf{t}_{\mathrm{o}}\right)-\xi_{1}^{\prime}\left(\mathbf{t}_{\mathrm{o}}\right)
$$

(it also directly follows from the constraint $W W^{\dagger}=1$ for the dressing operator). Plugging it back to (4.23), we can rewrite this equation in the form

$$
w^{\prime}\left(\mathbf{t}_{\mathrm{o}}, a\right) w\left(\mathbf{t}_{\mathrm{o}}-2\left[a^{-1}\right]_{\mathrm{o}},-a\right)=a f\left(\mathbf{t}_{\mathrm{o}}, a\right)\left(f\left(\mathbf{t}_{\mathrm{o}}, a\right)-1\right)+\frac{1}{2} f^{\prime}\left(\mathbf{t}_{\mathrm{o}}, a\right) .
$$


Using (4.11), we conclude that

$$
\begin{aligned}
& \partial_{x} \log w\left(\mathbf{t}_{\mathrm{o}}, a\right)=a\left(f\left(\mathbf{t}_{\mathrm{o}}, a\right)-1\right)+\frac{1}{2} \partial_{x} \log f\left(\mathbf{t}_{\mathrm{o}}, a\right) \\
& =\frac{1}{2}\left(\xi_{1}\left(\mathbf{t}_{\mathrm{o}}\right)-\xi_{1}\left(\mathbf{t}_{\mathrm{o}}-2\left[a^{-1}\right]_{\mathrm{o}}\right)\right)+\frac{1}{2} \partial_{x} \log f\left(\mathbf{t}_{\mathrm{o}}, a\right) .
\end{aligned}
$$

Now, setting $\xi_{1}\left(x, \mathbf{t}_{\mathrm{o}}\right)=-2 \partial_{x} \log \tau\left(x, \mathbf{t}_{\mathrm{o}}\right)$ with some function $\tau\left(x, \mathbf{t}_{\mathrm{o}}\right)$ and integrating, we arrive at (4.9) with $G=f^{1 / 2}$ up to a common multiplier which does not depend on $x$.

The function $u$ in (4.3) can be also expressed through the tau-function. It is a matter of direct verification that the result of the action of the operator $\partial_{x}^{3}+6 u \partial_{x}+3 u^{\prime}-\partial_{t_{3}}$ to the wave function $\Psi$ of the form (4.7) is $O\left(z^{-1}\right)$ as $z \rightarrow \infty$, i.e.,

$$
\left(\partial_{x}^{3}+6 u \partial_{x}+3 u^{\prime}-\partial_{t_{3}}\right) \Psi=O\left(z^{-1}\right) e^{x z+\xi\left(\mathbf{t}_{0}, z\right)}
$$

if the conditions

$$
u=-\frac{1}{2} \xi_{1}^{\prime}, \quad u^{\prime}=\xi_{1} \xi_{1}^{\prime}-\xi_{1}^{\prime \prime}-\xi_{2}^{\prime}
$$

hold true. Since from (4.9) it follows that

$$
\xi_{1}=-2 \partial_{x} \log \tau, \quad \xi_{2}=2\left(\partial_{x} \log \tau\right)^{2}+\partial_{x}^{2} \log \tau,
$$

we have

$$
u=\partial_{x}^{2} \log \tau
$$

and the second equality in (4.28) holds identically.

\subsection{The equation for the tau-function}

As it follows from (4.8), (4.9), the CKP hierarchy is equivalent to the following relation for the tau-function:

$$
\oint_{C_{\infty}} e^{\xi\left(\mathbf{t}_{\mathrm{o}}-\mathbf{t}_{\mathrm{o}}^{\prime}, z\right)} \tau\left(x, \mathbf{t}_{\mathrm{o}}-2\left[z^{-1}\right]_{\mathrm{o}}\right) \tau\left(x, \mathbf{t}_{\mathrm{o}}^{\prime}+2\left[z^{-1}\right]_{\mathrm{o}}\right) G\left(x, \mathbf{t}_{\mathrm{o}}, z\right) G\left(x, \mathbf{t}_{\mathrm{o}}^{\prime},-z\right) \frac{d z}{2 \pi i}=0
$$

valid for all $\mathbf{t}_{\mathrm{o}}, \mathbf{t}_{\mathrm{o}}^{\prime}$. Set $\mathbf{t}_{\mathrm{o}}^{\prime}=\mathbf{t}_{\mathrm{o}}-2\left[a^{-1}\right]_{\mathrm{o}}-2\left[b^{-1}\right]_{\mathrm{o}}-2\left[c^{-1}\right]_{\mathrm{o}}$, then

$$
e^{\xi\left(\mathbf{t}_{o}-\mathbf{t}_{\mathrm{o}}^{\prime}, z\right)}=\frac{(a+z)(b+z)(c+z)}{(a-z)(b-z)(c-z)}
$$

and the residue calculus in (4.30) gives the following equation:

$$
\begin{gathered}
a(a+b)(a+c)(b-c) \tau^{[a]_{\circ}} \tau^{[b c]_{\circ}} G(-a) G^{[a b c]_{\circ}}(a) \\
+b(b+a)(b+c)(c-a) \tau^{[b]_{\circ}} \tau^{[a c]_{\circ}} G(-b) G^{[a b c]_{\circ}}(b) \\
+c(c+a)(c+b)(a-b) \tau^{[c]_{\circ}} \tau^{[a b]_{\circ}} G(-c) G^{[a b c]_{\circ}}(c) \\
+(a+b+c)(a-b)(b-c)(c-a) \tau \tau^{[a b c]_{\circ}} \\
+(a-b)(b-c)(c-a)\left(\tau^{[a b c]_{\circ}} \partial_{t_{1}} \tau-\tau \partial_{t_{1}} \tau^{[a b c]_{\circ}}\right)=0,
\end{gathered}
$$


where $\tau^{[a]_{\mathrm{o}}}=\tau\left(x, \mathbf{t}_{\mathrm{o}}+2\left[a^{-1}\right]_{\mathrm{o}}\right), \tau^{[a b]_{\mathrm{o}}}=\tau\left(x, \mathbf{t}_{\mathrm{o}}+2\left[a^{-1}\right]_{\mathrm{o}}+2\left[b^{-1}\right]_{\mathrm{o}}\right), G^{[a]_{\mathrm{o}}}(z)=G\left(x, \mathbf{t}_{\mathrm{o}}+\right.$ $\left.2\left[a^{-1}\right]_{\mathrm{o}}, z\right)$ and so on. Equation (4.31) should be valid for all $a, b, c$. Let us tend $c$ to infinity. The highest terms proportional to $c^{3}$ vanish identically. The terms of order $c^{2}$ give the equation

$$
\frac{a+b}{a-b}\left(a G(-a) G^{[a b]_{\mathrm{o}}}(a)-b G(-b) G^{[a b]_{\mathrm{o}}}(b)\right)=\left(a+b-\partial_{t_{1}} \log \frac{\tau^{[a b]_{\mathrm{o}}}}{\tau}\right) \frac{\tau \tau^{[a b]_{\mathrm{o}}}}{\tau^{[a]_{\mathrm{o}}} \tau^{[b]_{\mathrm{o}}}},
$$

or, in the more detailed notation,

$$
\begin{gathered}
\frac{z_{1}+z_{2}}{z_{1}-z_{2}}\left[\left(z_{1}-\partial_{t_{1}} \log \frac{\tau^{\left[z_{1} z_{2}\right]_{\mathrm{o}}}}{\tau^{\left[z_{2}\right]_{\mathrm{o}}}}\right)^{1 / 2}\left(z_{1}-\partial_{t_{1}} \log \frac{\tau^{\left[z_{1}\right]_{\mathrm{o}}}}{\tau}\right)^{1 / 2}\right. \\
\left.-\left(z_{2}-\partial_{t_{1}} \log \frac{\tau^{\left[z_{1} z_{2}\right]_{\mathrm{o}}}}{\tau^{\left[z_{1}\right]_{\mathrm{o}}}}\right)^{1 / 2}\left(z_{2}-\partial_{t_{1}} \log \frac{\tau^{\left[z_{2}\right]_{\mathrm{o}}}}{\tau}\right)^{1 / 2}\right] \\
=\left(z_{1}+z_{2}-\partial_{t_{1}} \log \frac{\tau^{\left[z_{1} z_{2}\right]_{\mathrm{o}}}}{\tau}\right) \frac{\tau \tau^{\left[z_{1} z_{2}\right]_{\mathrm{o}}}}{\tau^{\left[z_{1}\right]_{\mathrm{o}}} \tau^{\left[z_{2}\right]_{\mathrm{o}}}} .
\end{gathered}
$$

This is the equation for the tau-function of the CKP hierarchy. It should hold for all $z_{1}, z_{2}$. In contrast to the cases of the KP and BKP hierarchies, this equation is not bilinear.

\subsection{The CKP hierarchy and the KP hierarchy}

Here we give a characterization of those KP tau-functions which correspond to solutions of the CKP hierarchy and show that the CKP tau-function is the square root of the KP one.

Comparing (2.13) and (4.8), we conclude that the wave functions of the CKP and KP hierarchies are related as

$$
\begin{aligned}
& \Psi\left(x, \mathbf{t}_{\mathrm{o}}, z\right)=e^{\chi(z)} \Psi^{\mathrm{KP}}\left(x, t_{1}, 0, t_{3}, 0, \ldots, z\right), \\
& \Psi\left(x, \mathbf{t}_{\mathrm{o}},-z\right)=e^{-\chi(z)} \Psi^{\dagger \mathrm{KP}}\left(x, t_{1}, 0, t_{3}, 0, \ldots, z\right) .
\end{aligned}
$$

Here $\chi(z)$ is some function such that $\chi(\infty)=0$, i.e.

$$
\Psi^{\dagger \mathrm{KP}}\left(x, t_{1}, 0, t_{3}, 0, \ldots, z\right)=e^{2 \chi_{\mathrm{e}}(z)} \Psi^{\mathrm{KP}}\left(x, t_{1}, 0, t_{3}, 0, \ldots,-z\right),
$$

where $\chi_{\mathrm{e}}(z)=\frac{1}{2}(\chi(z)+\chi(-z))$ is the even part of the function $\chi(z)$. From (2.11), (2.12) and (4.34) it follows that the KP tau-function is the extension of a solution of the CKP hierarchy if and only if the equation

$$
\begin{aligned}
\tau^{\mathrm{KP}}\left(x, t_{1}+z^{-1}\right. & \left., \frac{1}{2} z^{-2}, t_{3}+\frac{1}{3} z^{-3}, \frac{1}{4} z^{-4}, \ldots\right) \\
& =e^{2 \chi_{\mathrm{e}}(z)} \tau^{\mathrm{KP}}\left(x, t_{1}+z^{-1},-\frac{1}{2} z^{-2}, t_{3}+\frac{1}{3} z^{-3},-\frac{1}{4} z^{-4}, \ldots\right)
\end{aligned}
$$

holds identically for all $z, x, t_{1}, t_{3}, t_{5}, \ldots$. Shifting the odd times, we can rewrite this condition as

$$
\log \tau^{\mathrm{KP}}\left(x, t_{1}, \frac{1}{2} z^{-2}, t_{3}, \frac{1}{4} z^{-4}, \ldots\right)-\log \tau^{\mathrm{KP}}\left(x, t_{1},-\frac{1}{2} z^{-2}, t_{3},-\frac{1}{4} z^{-4}, \ldots\right)=2 \chi_{\mathrm{e}}(z) .
$$


Comparing the coefficients at $z^{-2}$ of the expansions of the left and right hand sides of (4.36) and passing to an equivalent tau-function if necessary, we get the condition

$$
\left.\partial_{t_{2}} \log \tau^{\mathrm{KP}}\right|_{t_{2 k}=0}=0, \quad k \geq 1
$$

It is the CKP counterpart of the condition (3.39) specific for the BKP hierarchy. In [11] it is proved that this is also a sufficient condition that the tau-function $\tau^{\mathrm{KP}}$ generates a solution to the CKP hierarchy. The proof is based on the technique developed in [17, 18, 19].

Let us introduce the auxiliary wave function $\psi$ in the same way as for the BKP hierarchy:

$$
\psi=e^{x z+\xi\left(\mathbf{t}_{\mathrm{o}}, z\right)} \frac{\tau\left(x, \mathbf{t}_{\mathrm{o}}-2\left[z^{-1}\right]_{\mathrm{o}}\right)}{\tau\left(x, \mathbf{t}_{\mathrm{o}}\right)},
$$

then the wave function (4.9) is

$$
\Psi=z^{-1 / 2} \sqrt{\partial_{x} \log \psi} \cdot \psi=(2 z)^{-1 / 2} \sqrt{\partial_{x} \psi^{2}} .
$$

We will prove that the CKP and KP tau-functions are related as

$$
\tau=\sqrt{\tau^{\mathrm{KP}}}
$$

The tau-function $\tau\left(x, \mathbf{t}_{\mathrm{o}}\right)$ has square root singularities in $x$. However, it appears that the expression $\partial_{x} \psi^{2}$ is a full square for all $z$, i.e., $\sqrt{\partial_{x} \psi^{2}}$ and, therefore, $\Psi$ is an entire function of $x$ and $\mathbf{t}_{\mathrm{o}}$ (no square root singularities!). This is similar to the fact that for the BKP hierarchy the function $\tau^{\mathrm{KP}}$ is a full square. The bilinear identity (4.8) acquires the form

$$
\oint_{C_{\infty}} \sqrt{\partial_{x} \psi^{2}\left(x, \mathbf{t}_{\mathrm{o}}, z\right)} \sqrt{\partial_{x} \psi^{2}\left(x, \mathbf{t}_{\mathrm{o}}^{\prime},-z\right)} \frac{d z}{2 \pi i z}=0
$$

In order to prove that $\tau=\sqrt{\tau^{\mathrm{KP}}}$ (see [9]) we compare two expressions for the wave function $\Psi$ of the CKP hierarchy. The first one is in terms of the KP tau-function,

$$
\Psi^{\mathrm{KP}}=e^{x z+\xi\left(\mathbf{t}_{\mathrm{o}}, z\right)} \frac{\tau^{\mathrm{KP}}\left(x, t_{1}-z^{-1},-\frac{1}{2} z^{-2}, t_{3}-\frac{1}{3} z^{-3},-\frac{1}{4} z^{-4}, \ldots\right)}{\tau^{\mathrm{KP}}\left(t_{1}, 0, t_{3}, 0, \ldots\right)},
$$

and the second one (4.9) is in terms of the CKP tau-function $\tau$. Comparing (4.39) and (4.42), we get the equation

$$
\frac{1}{2 z} \partial_{x}\left(e^{2 x z} \frac{\tau^{2}\left(x, \mathbf{t}_{\mathrm{o}}-2\left[z^{-1}\right]_{\mathrm{o}}\right)}{\tau^{2}\left(x, \mathbf{t}_{\mathrm{o}}\right)}\right)=e^{2 x z}\left(\frac{\tau^{\mathrm{KP}}\left(x, \dot{\mathbf{t}}-\left[z^{-1}\right]\right)}{\tau^{\mathrm{KP}}(x, \dot{\mathbf{t}})}\right)^{2},
$$

where we again use the short-hand notation $\dot{\mathbf{t}}=\left\{t_{1}, 0, t_{3}, 0, \ldots\right\}$. Then we get that (4.43) is equivalent to the differential equation $\partial_{x} \varphi=-2 z \varphi$, where

$$
\varphi=\frac{\tau^{2}\left(x, \mathbf{t}_{\mathrm{o}}-2\left[z^{-1}\right]_{\mathrm{o}}\right)}{\tau^{2}\left(x, \mathbf{t}_{\mathrm{o}}\right)}-\frac{\tau^{\mathrm{KP}}\left(x, \dot{\mathbf{t}}-2\left[z^{-1}\right]_{\mathrm{o}}\right)}{\tau^{\mathrm{KP}}(x, \dot{\mathbf{t}})} .
$$

In (4.44), $\dot{\mathbf{t}}-2\left[z^{-1}\right]_{\mathrm{o}}=\left\{t_{1}-2 z^{-1}, 0, t_{3}-\frac{2}{3} z^{-3}, 0, \ldots\right\}$. The general solution of the differential equation is $\varphi=c\left(z, t_{3}, t_{5}, \ldots\right) e^{-2\left(x+t_{1}\right) z}$ but from (4.44) it follows that $\varphi$ is 
expanded in a power series as $\varphi=\varphi_{1} z^{-1}+\varphi_{2} z^{-2}+\ldots$ as $z \rightarrow \infty$, and this means that $c$ must be equal to 0 . Therefore, $\varphi=0$, i.e.

$$
\frac{\tau^{2}\left(x, \mathbf{t}_{\mathrm{o}}-2\left[z^{-1}\right]_{\mathrm{o}}\right)}{\tau^{2}\left(x, \mathbf{t}_{\mathrm{o}}\right)}=\frac{\tau^{\mathrm{KP}}\left(x, \dot{\mathbf{t}}-2\left[z^{-1}\right]_{\mathrm{o}}\right)}{\tau^{\mathrm{KP}}(x, \dot{\mathbf{t}})}
$$

for all $z$. This is an identity on solutions to the KP/CKP hierarchies. It follows from (4.45) that $\tau^{\mathrm{KP}}=$ const $\cdot \tau^{2}$, i.e. $\tau\left(x, \mathbf{t}_{\mathrm{o}}\right)=\sqrt{\tau^{\mathrm{KP}}(x, \dot{\mathbf{t}})}$ is a tau-function of the CKP hierarchy.

\subsection{Examples: soliton solutions}

\subsubsection{One-soliton solution}

We start from the simplest example of one-soliton solution. The tau-function for one CKP soliton is the square root of a specialization of 2-soliton tau-function of the KP hierarchy. The latter has the form

$$
\begin{gathered}
\tau^{\mathrm{KP}}(x, \mathbf{t})=1+\alpha \exp \left((p+q) x+\sum_{k \geq 1}\left(p^{k}-(-q)^{k}\right) t_{k}\right) \\
+\alpha \exp \left((p+q) x+\sum_{k \geq 1}\left(q^{k}-(-p)^{k}\right) t_{k}\right) \\
-\frac{\alpha^{2}(p-q)^{2}}{4 p q} \exp \left(2(p+q) x+\sum_{k \geq 1}\left(p^{k}+q^{k}-(-p)^{k}-(-q)^{k}\right) t_{k}\right),
\end{gathered}
$$

where $\alpha, p, q$ are arbitrary parameters. When all even times are put equal to zero, $t_{2}=$ $t_{4}=\ldots=0$, this expression simplifies:

$$
\tau^{\mathrm{KP}}=1+2 \alpha w-\frac{\alpha^{2}(p-q)^{2}}{4 p q} w^{2},
$$

where

$$
w=e^{(p+q) x+\xi\left(\mathbf{t}_{\mathrm{o}}, p\right)+\xi\left(\mathbf{t}_{\mathrm{o}}, q\right)} .
$$

We see that the tau-function $\tau=\sqrt{\tau^{\mathrm{KP}}}$ has two square root singularities at the points $w=w_{ \pm}$

$$
w_{ \pm}= \pm \frac{2 \sqrt{p q}}{\alpha(\sqrt{p} \mp \sqrt{q})^{2}} .
$$

A direct calculation shows that $\partial_{x} \psi^{2}$ (where $\psi$ is given by (4.38)) for the solution (4.47) is a full square for all $z$ :

$$
\frac{\partial_{x} \psi^{2}}{2 z}=e^{2 x z+2 \xi\left(\mathbf{t}_{\mathrm{o}}, z\right)}\left(\frac{1+\frac{\alpha\left(2 z^{2}-p^{2}-q^{2}\right)}{(z+p)(z+q)} w-\frac{\alpha^{2}(p-q)^{2}}{4 p q} \frac{(z-p)(z-q)}{(z+p)(z+q)} w^{2}}{1+2 \alpha w-\frac{\alpha^{2}(p-q)^{2}}{4 p q} w^{2}}\right)^{2}
$$




$$
=\left(\Psi^{\mathrm{KP}}\left(x, t_{1}, 0, t_{3}, 0, \ldots, z\right)\right)^{2},
$$

where $\Psi^{\mathrm{KP}}$ is constructed from the KP tau-function $\tau^{\mathrm{KP}}$ (4.46) according to formula (2.11). Hence $(2 z)^{-1 / 2} \sqrt{\partial_{x} \psi^{2}}$ and, therefore, $\Psi$ does not have square root singularities in the variable $z$. The bilinear identity (4.8), which in the present case has the explicit form

$$
\begin{gathered}
\oint_{C_{\infty}} e^{\xi\left(\mathbf{t}_{\mathrm{o}}, z\right)-\xi\left(\mathbf{t}_{\mathrm{o}}^{\prime}, z\right)}\left(1+\frac{\alpha\left(2 z^{2}-p^{2}-q^{2}\right)}{(z+p)(z+q)} w-\frac{\alpha^{2}(p-q)^{2}}{4 p q} \frac{(z-p)(z-q)}{(z+p)(z+q)} w^{2}\right) \\
\times\left(1+\frac{\alpha\left(2 z^{2}-p^{2}-q^{2}\right)}{(z-p)(z-q)} w^{\prime}-\frac{\alpha^{2}(p-q)^{2}}{4 p q} \frac{(z+p)(z+q)}{(z-p)(z-q)} w^{\prime 2}\right) \frac{d z}{2 \pi i}=0,
\end{gathered}
$$

can be checked directly by the residue calculus. (Here $w^{\prime}=e^{(p+q) x+\xi\left(\mathbf{t}_{\mathrm{o}}^{\prime}, p\right)+\xi\left(\mathbf{t}_{\mathrm{o}}^{\prime}, q\right)}$.)

\subsubsection{Multi-soliton solutions}

The general KP tau-function for $M$-soliton solution has $3 M$ arbitrary parameters $\alpha_{i}, p_{i}$, $q_{i}(i=1, \ldots, M)$ and is given by equation (3.46). We denote this tau-function as

$$
\tau^{\mathrm{KP}}\left[\begin{array}{c}
\alpha_{1} \\
p_{1}, q_{1}
\end{array} ; \begin{array}{c}
\alpha_{2}, q_{2} \\
p_{2}, \alpha_{3}, q_{3}
\end{array} ; \begin{array}{c}
\alpha_{4} \\
p_{4}, q_{4}
\end{array} ; \cdots ; \begin{array}{ccc}
\alpha_{2 N-1} & \alpha_{2 N} \\
p_{M-1}, q_{M-1} & p_{M}, q_{M}
\end{array}\right] .
$$

The parameters $p_{i}, q_{i}$ are sometimes called momenta of solitons.

The multi-soliton tau-function of the CKP hierarchy is the square root of the $\tau^{\mathrm{KP}}$ specialized as

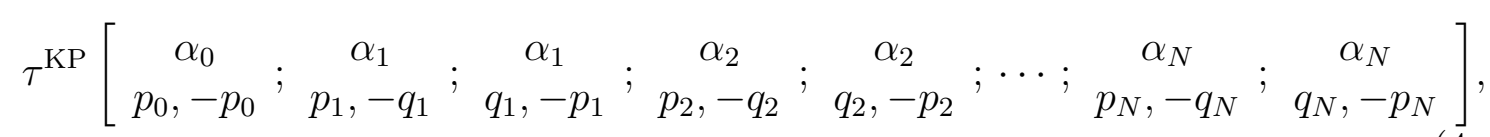

where it is implied that even times evolution is suppressed $\left(t_{2 k}=0\right.$ for all $\left.k \geq 1\right)$. Clearly, the total number of independent parameters is $3 N+2$. If $\alpha_{0}=0$, the tau-function (3.47) reduces to

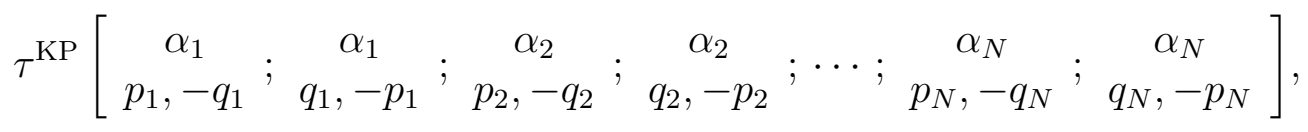

and it is this tau-function which is usually called the $N$-soliton CKP tau-function in the literature (see, e.g. [3]). It is a specialization of $2 N$-soliton KP tau-function and has $3 N$ free parameters.

\section{Dispersionless limit of the BKP and CKP hierar- chies}

The dispersionless limit is the limit $\hbar \rightarrow 0$ after the substitutions

$$
t_{k} \rightarrow t_{k} / \hbar, \quad \tau=\exp \left(F / \hbar^{2}\right)
$$


Making these substitutions in (3.32) and performing the limit, we get the equation

$$
\frac{p\left(z_{1}\right)-p\left(z_{2}\right)}{z_{1}-z_{2}}=\frac{p\left(z_{1}\right)+p\left(z_{2}\right)}{z_{1}+z_{2}} e^{4 D^{\circ}\left(z_{1}\right) D^{\circ}\left(z_{2}\right) F}
$$

where

$$
p(z)=z-2 \partial_{t_{1}} D^{\circ}(z) F
$$

and $D^{\circ}(z)$ is the differential operator

$$
D^{\mathrm{o}}(z)=\sum_{k \geq 1, \text { odd }} \frac{z^{-k}}{k} \partial_{t_{k}} .
$$

The (odd) function $p(z)$ has the expansion

$$
p(z)=z-\frac{u}{z}+\sum_{k \geq 3, \text { odd }} u_{k} z^{-k}
$$

where

$$
u=2 \partial_{t_{1}}^{2} F .
$$

Equation (5.1) is the generating equation for the dispersionless BKP (dBKP) hierarchy in the Hirota form [22, 23, 24]. It is remarkable that the dispersionless limit of the CKP equation (4.33) is the same, so the dispersionless limits of the BKP and CKP hierarchies coincide.

Let us show how to represent the dispersionless hierarchy in the Lax form. Taking logarithm of equation (5.1), differentiating with respect to $t_{1}$ and using the definition (5.4), we obtain the equation

$$
2 D^{\mathrm{o}}\left(z_{1}\right) p\left(z_{2}\right)=\partial_{t_{1}} \log \frac{p\left(z_{1}\right)+p\left(z_{2}\right)}{p\left(z_{1}\right)-p\left(z_{2}\right)}
$$

from which it follows that $D^{\circ}\left(z_{1}\right) p\left(z_{2}\right)=D^{\circ}\left(z_{2}\right) p\left(z_{1}\right)$ (this follows also from the definition (5.4)). Tending $z_{2} \rightarrow \infty$, we get

$$
\partial_{t_{1}} p(z)=-D^{\mathrm{o}}(z) u
$$

The next step is to rewrite equation (3.11) in terms of the function $z(p)$, inverse to the $p(z)$ (like $p(z)$, it is an odd function with the Laurent series of the form $z(p)=p+O\left(p^{-1}\right)$ ). Using the relation

$$
\partial_{t_{k}} p(z)=-\frac{\partial_{t_{k}} z(p)}{\partial_{p} z(p)}, \quad k \geq 1
$$

we get, after simple transformations:

$$
2 D^{\mathrm{o}}\left(z_{1}\right) z(p)=\left\{z(p), \log \frac{p\left(z_{1}\right)-p}{p\left(z_{1}\right)+p}\right\},
$$

where

$$
\{f, g\}:=\frac{\partial f}{\partial t_{1}} \frac{\partial g}{\partial p}-\frac{\partial g}{\partial t_{1}} \frac{\partial f}{\partial p}
$$


is the Poisson bracket. This is the generating Lax equation for the dBKP hierarchy, $z(p)$ being the Lax function (the dispersionless limit of the Lax operator (3.1)). Expanding equation (5.9) in powers of $z_{1}$, one obtains the hierarchy of Lax equations through the Faber polynomials $\mathcal{B}_{k}(p)$ introduced by the expansion

$$
-\log \frac{p(z)-p}{z}=\sum_{k \geq 1} \frac{z^{-k}}{k} \mathcal{B}_{k}(p) .
$$

For example, $\mathcal{B}_{1}(p)=p$. It is easy to see that

$$
\mathcal{B}_{k}(p)=\left(z^{k}(p)\right)_{\geq 0},
$$

where $(\ldots)_{\geq 0}$ is the polynomial part of the Laurent series in $p$ (containing only nonnegative powers of the variable $p$ ). The fact that $p(z)$ is an odd function implies that $\mathcal{B}_{k}(-p)=(-1)^{k} \mathcal{B}_{k}(p)$ and we have the expansion

$$
\log \frac{p(z)+p}{p(z)-p}=2 \sum_{k \geq 1, \text { odd }} \frac{z^{-k}}{k} \mathcal{B}_{k}(p) .
$$

The Lax equations are of the form

$$
\partial_{t_{k}} z(p)=\left\{\mathcal{B}_{k}(p), z(p)\right\}=\left\{\left(z^{k}(p)\right)_{\geq 0}, z(p)\right\}, \quad k \text { odd } .
$$

\section{Acknowledgments}

The author thanks V. Akhmedova, I. Krichever, S. Natanzon and D. Rudneva for discussions. This work was supported by the Russian Science Foundation under grant 19-11-00275.

\section{References}

[1] E. Date, M. Jimbo, M. Kashiwara and T. Miwa, Transformation groups for soliton equations: Nonlinear integrable systems - classical theory and quantum theory (Kyoto, 1981). Singapore: World Scientific, 1983, 39-119.

[2] E. Date, M. Jimbo, M. Kashiwara and T. Miwa, Transformation groups for soliton equations IV. A new hierarchy of soliton equations of KP type, Physica D 4D (1982) 343-365.

[3] E. Date, M. Jimbo, M. Kashiwara and T. Miwa, KP hierarchy of orthogonal and symplectic type - Transformation groups for soliton equations VI, J. Phys. Soc. Japan 50 (1981) 3813-3818.

[4] M. Jimbo and T. Miwa, Soliton equations and infinite dimensional Lie algebras, Publ. RIMS, Kyoto University 19 (1983) 943-1001.

[5] I. Loris and R. Willox, Symmetry reductions of the BKP hierarchy, Journal of Mathematical Physics 40 (1999) 1420-1431. 
[6] M.-H. Tu, On the BKP Hierarchy: Additional Symmetries, Fay Identity and AdlerShiota-van Moerbeke Formula, Letters in Mathematical Physics 81 (2007) 93-105.

[7] A. Dimakis and F. Müller-Hoissen, BKP and CKP revisited: the odd KP system, Inverse Problems 25 (2009) 045001, arXiv:0810.0757.

[8] L. Chang and C.-Z. Wu, Tau function of the CKP hierarchy and non-linearizable Virasoro symmetries, Nonlinearity 26 (2013) 2577-2596.

[9] J. Cheng and J. He, The "ghost" symmetry in the CKP hierarchy, Journal of Geometry and Physics, 80 (2014) 49-57.

[10] J. van de Leur, A. Orlov and T. Shiota, CKP hierarchy, bosonic tau function and bosonization formulae, SIGMA 8 (2012) 036, arXiv:1102.0087

[11] I. Krichever and A. Zabrodin, Kadomtsev-Petviashvili turning points and CKP hierarchy, preprint arXiv:2012.04482.

[12] I. Krichever, Methods of algebraic geometry in the theory of non-linear equations, Russian Math. Surveys 32 (1977) 185-213.

[13] E. Date, M. Jimbo, M. Kashiwara and T. Miwa, Quasi-periodic solutions of the orthogonal KP equation - Transformation groups of soliton equations V, Publ. RIMS, Kyoto Univ. 18 (1982) 1111-1119.

[14] S. Natanzon, Differential equations for Prym theta-functions. The criteria of reality of two-dimensional finite-gap potential Schrödinger operators, Funk. Anal. i ego Pril. 26 (1992) 17-26.

[15] D. Rudneva and A. Zabrodin, Dynamics of poles of elliptic solutions to BKP equation, Journal of Physics A: Math. Theor. 53 (2020) 075202.

[16] A. Zabrodin, Elliptic solutions to integrable nonlinear equations and many-body systems, Journal of Geometry and Physics 146 (2019) 103506.

[17] S. Natanzon, Formulas for $A_{n}$ and $B_{n}$-solutions of WDVV equations, J. Geom. Phys. 39 (2001) 323-336.

[18] S. Natanzon, Witten solution for the Gelfand-Dikii hierarchy, Funk. Anal. i ego Prilozh. 37:1 (2003) 25-37 (English translation: Func. Anal. Appl. 37:1 (2003) 2131).

[19] S. Natanzon and A. Zabrodin, Formal solution to the KP hierarchy, J. Phys. A: Math. Theor. 49 (2016) 145206.

[20] T. Takebe, From general Zakharov-Shabat equations to the KP and the Toda lattice hierarchies, Int. J. Mod. Phys. A 7 (1992) No. supp01b, 923-939.

[21] K. Ueno and K. Takasaki, Toda lattice hierarchy, Advanced Studies in Pure Mathematics 4 (1984) 1-95.

[22] K. Takasaki, Quasi-classical limit of BKP hierarchy and W-infinity symmetries, Lett. Math. Phys. 28 (1993) 177-185. 
[23] K. Takasaki, Dispersionless Hirota equations of two-component BKP hierarchy, SIGMA 2 (2006) 057.

[24] T. Takebe, Dispersionless BKP hierarchy and quadrant Löwner equation, SIGMA 10 (2014) 023. 